\title{
Strong resolution of singularities in characteristic zero
}

\author{
Santiago Encinas and Herwig Hauser
}

\begin{abstract}
We present a concise proof for the existence and construction of a strong resolution of excellent schemes of finite type over a field of characteristic zero. Our proof is based on earlier work of Villamayor, Encinas-Villamayor and Bierstone-Milman. It proposes some substantial simplifications which may be helpful for a better understanding of how to prove Hironaka's famous theorem on embedded resolution of singularities.
\end{abstract}

Mathematics Subject Classification (2000). 14E15, 14B05, 32S05, 32S10, 32S45.

Keywords. Resolution of singularities.

Hironaka's spectacular proof of resolution of singularities is built on a multiple and intricate induction argument. It is so involved that only few people could really understand it. The constructive proofs given later by Villamayor, Bierstone-Milman and Encinas-Villamayor presented important steps towards a better understanding of the reasoning. They describe an algorithmic procedure for resolution, using a local invariant to show that the situation improves under blowup. The centers of blowup are given as the locus where the invariant attains its maximal value.

Despite this simple outset, the proofs are not easy, mostly because the definition of the invariant involves the whole sequence of blowups having occurred so far in the resolution process. To make the invariant intrinsic and to patch the local constructions various equivalence relations have to be introduced.

When trying to understand these proofs it became clear that to define the invariant it is actually not necessary to refer to the entire sequence of earlier blowups. It is sufficient to have information on two sets of exceptional components at each stage of the process. Including this information to the resolution datum (called mobile in this paper) the invariant can be defined directly without going back to the very beginning of the resolution process.

"If you wish to cross the Sahara, better take a map with you where you mark daily the trajectory you have made so far rather than to return every morning to the starting point in order to know in which direction to continue."

Already Abhyankar and Hironaka took a map with them, and the idea is also 
used in the other papers, though in a different way. The extra luggage we shall carry with us in this paper specifies exactly the combinatorial book-keeping information which allows to construct the local invariant. The resulting proof should be regarded as a conceptualized version of the existing proofs. In substance it is the same proof.

Although the invariant can now be defined promptly potential readers may still hesitate to try to understand how to prove resolution of singularities. For many, it is a black box better not to be touched. But Hironaka's proof is a phantastic proof, and very beautiful. Our objective is to transmit this fascination - and to open the box. So let us start.

\section{The result}

Let $X$ be a reduced singular scheme. A strong resolution of $X$ is, for every closed embedding of $X$ into a regular ambient scheme $W$, a proper birational morphism $\varepsilon$ from a regular scheme $W^{\prime}$ onto $W$ subject to the following conditions.

Explicitness. $\varepsilon$ is a composition of blowups of $W$ in regular closed centers $Z$ transversal to the exceptional loci.

Embeddedness. The strict transform $X^{\prime}$ of $X$ is regular and has normal crossings with the exceptional locus in $W^{\prime}$.

Excision. The morphism $X^{\prime} \rightarrow X$ does not depend on the embedding of $X$ in $W$.

Equivariance. $\varepsilon$ commutes with smooth morphisms $W^{-} \rightarrow W$, embeddings $W \rightarrow W^{+}$, and separable field extensions. In particular, $\varepsilon$ is an isomorphism outside the singular locus of $X$ and commutes with group actions.

The resolution of $X$ is global with centers equal to the top locus of an upper semicontinuous invariant $i_{a}(X)$ on $W$ given by the local rings of $X$ (effectiveness). The resolution commutes with open immersions, local and global diffeomorphisms and taking cartesian products with regular schemes. The smooth morphisms of equivariance need not be defined over the ground field. Passage to the completions implies resolution of formal schemes. The resolution process can be implemented.

The existence of resolutions satisfying the first two properties was established for excellent schemes of finite type over a field of characteristic zero by Hironaka [H1]. For such schemes, the construction of a strong resolution in the above sense through a local invariant defining the centers is due to Villamayor [V1, V2], Bierstone-Milman [BM1, BM2, BM3] and Encinas-Villamayor [EV1, EV2]. The algorithm was implemented by Bodnár-Schicho [BS].

Weak resolution theorems in characteristic zero have been established with different methods by Abramovich-de Jong, Abramovich-Wang and BogomolovPantev [AJ, AW, BP]. The main results on resolution in positive characteristic are due to Abhyankar, Giraud, Lipman, Cossart, de Jong and Kuhlmann. We refer to the survey [Ha1] for details. 
The proof of the present paper for the existence and construction of strong resolutions in characteristic zero relies on ideas and techniques from Hironaka, Abhyankar and Giraud. The invariant and the centers are almost identical to the ones used by Villamayor, Bierstone-Milman and Encinas-Villamayor. There are, however, several improvements with respect to the existing literature. These are listed below.

The resolution of schemes will be deduced from the resolution of singular mobiles. Mobiles are intrinsic global data which encode the singular and combinatorial structure of the resolution problem and record its transversality with the exceptional divisor. Locally, each mobile is exploited through the choice of a punctual setup associated to it. This is a string of ideals in decreasing dimensions which determines the resolution invariant. The latter is shown not to depend on the chosen setup. It thus gives a local measure of the resolution complexity of the mobile. Its top locus defines a global center of blowup, which in turn determines the transform of the mobile. The invariant of the transformed mobile is shown to have decreased and thus induction applies to give the resolution of mobiles and hence of schemes.

The main issues of the proof are the following.

- Coverings of global and patchings of local objects are avoided by considering stratified ideals and punctual setups of mobiles defined only locally. This and the use of transversal handicaps eliminates also the use of equivalence relations. - All constructions and arguments but one are characteristic free, the exception being the existence of osculating hypersurfaces.

- The Hilbert-Samuel function and normal flatness are avoided by working with the order of ideals and weak transforms. The centers of blowup lie in the weak transforms of the scheme though possibly not in the strict transform. This is not a serious drawback, since all centers map to the singular locus, but simplifies things considerably. For equidimensional schemes, the HilbertSamuel function had already been eliminated by Encinas-Villamayor in [EV2] using a somewhat different argument; for the general case, see also [BV].

- The relevant information on the history of the resolution process is encoded in the combinatorial and transversal handicap of the mobile and its transforms. To define the invariant it is no longer necessary to consider the whole sequence of earlier blowups.

Standard results on the order of ideals and on hypersurfaces of maximal contact as well as some straightforward verifications are omitted. Paragraphs in small characters provide background information and/or proofs of well known or technical results appearing in the main body of the text. Superscripts refer to the appendix. For notational convenience there appear rational powers of ideals. These could be avoided by raising the ideals to suitable powers, as will be indicated in parentheses. As taking the order commutes with powers, the exponents are treated as integers.

The various constructions of the present paper are often justified only a posteriori through their role in the proof. This makes it hard to get a feeling for them 
at the moment when they are introduced. The expository paper [Ha2] provides these justifications $a b$ initio. It shows how the constructions arise naturally when trying to prove resolution of singularities from scratch.

We are indebted to Hironobu Maeda for very valuable references, and to Gábor Bodnár for many probing questions. Substantial improvements regarding the organization of the paper were suggested by a highly competent anonymous referee.

\section{Idea of proof}

Let $J$ be the ideal defining $X$ in $W$. We want to transform $J$ by a sequence of blowups into a simple form, i.e., so that the pull-back of $J$ becomes a monomial principal ideal. The resolution of $X$ will be then deduced from this monomialization. Let us place ourselves at a certain stage of the resolution process. We will have to decide on the center of the next blowup. The ideal $J$ will stem from earlier blowups, so that exceptional components can be factored from $J$ to a certain power. This factor will be noted down in what we call the combinatorial handicap. It is a (non-reduced) normal crossings divisor $D$ in $W$ supported by the current exceptional locus $F$ so that $J$ factors into $J=M \cdot I$ with $M$ the ideal defining $D$ in $W$, and some ideal $I$ of $W$ which is still unresolved. Our objective will be to lower the order $O$ of $I$ at the points of $W$ by further blowups, until $I$ becomes 1 and $J=M$ is the required monomial. A separate argument will show that the monomialization of ideals implies the resolution of singular schemes.

Fix the above situation. The center $Z$ of the next blowup $\pi: W^{\prime} \rightarrow W$ should be a closed and globally defined regular subscheme of $W$, which is transversal to the exceptional locus. In addition, we wish to have $Z$ inside the top locus of $I$, i.e., in the set of points where the order of $I$ in $W$ is maximal. In particular, $o=\operatorname{ord}_{a} I$ shall be constant for $a \in Z$. This will ensure that the order $o^{\prime}$ of the transform of $I$ under the blowup of $W$ with center $Z$ will not increase. Once $Z$ satisfies these two conditions, we will have $o^{\prime} \leq o$ for all points of the new exceptional component $Y^{\prime}=\pi^{-1}(Z)$ in $W^{\prime}$, and the total transform of $M$ will be an ideal $M^{*}$ defining again a normal crossings divisor in $W^{\prime}$. By construction, the total transform $J^{*}$ of $J$ will factor into $J^{*}=M^{*} \cdot I\left(Y^{\prime}\right)^{o} \cdot I^{\curlyvee}$, where $I^{\curlyvee}=I\left(Y^{\prime}\right)^{-o} \cdot I^{*}$ denotes the weak transform of $I$. Setting $J^{\prime}=J^{*}, M^{\prime}=M^{*} \cdot I\left(Y^{\prime}\right)^{o}$ and $I^{\prime}=I^{\curlyvee}$ we get again a product

$$
J^{\prime}=M^{\prime} \cdot I^{\prime}
$$

with prescribed exceptional factor $M^{\prime}=I_{W^{\prime}}\left(D^{\prime}\right)$ given by the transformed combinatorial handicap $D^{\prime}=D^{*}+o \cdot Y^{\prime}$, and new exceptional locus $F^{\prime}$. Thus our (preliminary) resolution datum, made precise later in the concept of mobiles, consists at each stage of a product of ideals $J=M \cdot I$ and two normal crossings divisors $D$ and $F$ in $W$.

We are left to determine a suitable center $Z$, and to show that at the points where equality $o^{\prime}=o$ holds the situation has improved. Both tasks will be accom- 
plished simultaneously by associating to $J, D$ and $F$ a local upper semicontinuous invariant $i_{a}(J)$. Its top locus will be the required center $Z$, and $i_{a}(J)$ will drop after blowup.

The crucial advantage in characteristic zero is that there exists locally at each point a regular hypersurface $V$ of $W$ whose successive transforms under any blowup with center inside $V$ contain all equiconstant points, i.e., the points where the orders $o^{\prime}, o^{\prime \prime}, \ldots$ of the transforms of $I$ remain constant (hypersurface of maximal contact). Choose such a $V$ locally at a point $a$ of $W$. Let $Z$ be any center of blowup inside $V$. As the transform $V^{\prime}$ of $V$ contains the points $a^{\prime}$ above $a$ where $o^{\prime}=o$ it suffices to compare $J$ and $J^{\prime}$ at points of $V$ and $V^{\prime}$, i.e., inside hypersurfaces.

The idea then is to associate to $J=M \cdot I$ and $J^{\prime}=M^{\prime} \cdot I^{\prime}$ ideals $J_{-}$and $\left(J^{\prime}\right)_{-}$in $V$ and $V^{\prime}$ which reveal the expected improvement. Once we have constructed the appropriate ideal $J_{-}$we can apply induction on the dimension to find the center and the invariant, since $J_{-}$lives in an ambient space of smaller dimension. In this way we may assume that we have already constructed a local upper semicontinuous invariant $i_{a}\left(J_{-}\right)$whose top locus prescribes a regular center $Z_{-}$in $V$ such that blowing up $V$ in $Z_{-}$makes $i_{a}\left(J_{-}\right)$drop (except if $J_{-}$is already resolved). If $i_{a}\left(J_{-}\right)$ does not depend on any choices (in particular, not on the local choice of $V$ ), the center will automatically be defined globally. We will give the definition of $J_{-}$in a moment.

There arise two problems. The center $Z_{-}$associated to $J_{-}$may not be transversal to the exceptional locus $F$, and the transform of $J_{-}$under the blowup $V^{\prime} \rightarrow V$ with center $Z_{-}$may not coincide with the ideal $\left(J^{\prime}\right)_{-}$associated to $J^{\prime}$ in $V^{\prime}$.

If $Z_{-}$is not transversal to $F$, we have to solve this subproblem first. Auxiliary blowups with smaller centers will make $Z_{-}$transversal to $F$, so that it can be really taken as center. Actually, $J_{-}$will be built up so that this subproblem is solved in parallel: we specify the components $E$ of $F$ to which $Z_{-}$may not be transversal, noted down in the transversal handicap, and then resolve the ideal $Q=I_{V}(E \cap V)$ in $V$ by auxiliary blowups. Once its weak transform has become 1 , $V$ and $E$ will be separated from each other, and transversality holds since $Z_{-} \subset V$. This separation cannot and need not be realized for the whole exceptional locus $F$ : the components of $F \backslash E$ will a priori be transversal to $Z_{-}$and therefore do not affect the transversality problem. Of course, the critical components $E$ inside $F$ have to be determined explicitly.

The second problem is handled by taking for $\left(J_{-}\right)^{\prime}$ an intermediate transform between total and weak transform (the controlled transform; it is given by a number $c$, the control). For this, the required commutativity $\left(J^{\prime}\right)_{-}=\left(J_{-}\right)^{\prime}$ is a check in local coordinates. It is here that we need to work with factorized ideals $J=M \cdot I$, because $J$ and $I$ will transform differently. The controlled transform of $J$ ensures commutativity while its order may increase, whereas the weak transform of $I$ would not yield commutativity while its order decreases or remains constant.

We see that everything concentrates on defining the correct ideal $J_{-}$. This will be achieved through the coefficient ideal of $I$ in $V$. It is obtained from $I$ by 
expanding its elements with respect to a local coordinate defining $V$ in $W$ and taking the ideal generated by (equilibrated powers of) its coefficients. To include the transversality problem, one has to take the coefficient ideal not of $I$ but of a product $I \cdot Q$ where $Q$ defines the possibly non-transversal components from $E$. Then $Z_{-}$will be contained in the top locus of $Q$, hence in all components of $E$. Therefore it will automatically be transversal to $F$.

It remains to define the invariant $i_{a}(J)$. It is given as the vector

$$
i_{a}(J)=\left(\operatorname{ord}_{a} I, \operatorname{ord}_{a} Q, m_{a}, i_{a}\left(J_{-}\right)\right),
$$

where the component $m_{a}$ is of combinatorial nature. It only becomes relevant when $J_{-}$is already resolved and its invariant $i_{a}\left(J_{-}\right)$cannot improve. The invariant is considered with respect to the lexicographic order. It depends on $J, D$ and $E$.

If $J_{-}$is not resolved, we may assume by induction on the dimension that $i_{a}\left(J_{-}\right)$ will improve when blowing up its top locus $Z_{-}$in $V$ (the case of dimension of $V$ equal 1 being trivial). Eventually, $\left(\operatorname{ord}_{a} I, \operatorname{ord}_{a} Q\right)$ must drop. When the second component $\operatorname{ord}_{a} Q$ drops, the transversality subproblem improves. After finitely many steps it is solved, $\operatorname{ord}_{a} Q=0$. Larger centers become permissible. As $i_{a}\left(J_{-}\right)$ continues to improve the first component $\operatorname{ord}_{a} I$ must also drop sometime. Now induction applies to prove that finitely many blowups yield $\operatorname{ord}_{a} I=0$, which signifies that $J=M$ is a monomial.

The above considerations show that we need in each dimension informations on the divisor $D$ formed by those exceptional components which can be factored from $J$ and on the divisor $E$ of exceptional components which may pose a transversality problem. Therefore the combinatorial and the transversal handicap appearing in mobiles will consist of strings $D_{n}, \ldots, D_{1}$ and $E_{n}, \ldots, E_{1}$ of (stratified) normal crossings divisors in $W$. They are global objects which do not depend on any local choices, and obey prescribed rules of transformations under blowup. Thus we can define the transform of a mobile under blowup, and its resolution. Mobiles are the maps we take with us on our trip through the Sahara: Every day we write down how the combinatorial and transversal handicap have transformed under the last blowup. The transformation rule for the handicaps will depend on the point of the blowup we are considering (i.e., on the value of the local invariant at this point). As the invariant is upper semicontinuous and hence induces a stratification by locally closed sets, we will get stratified divisors.

The descent in dimension via local ideals $J_{-}$depends on the choice of the hypersurface $V$ and yields strings of ideals $J_{n}, \ldots, J_{1}$ (with $J_{n}=J, J_{n-1}=J_{-}$) in local flags $W_{n} \supset \cdots \supset W_{1}$ of regular subschemes, called the setup of the mobile. Each ideal factors into $J_{i}=M_{i} \cdot I_{i}$ according to $M_{i}=I_{W_{i}}\left(D_{i} \cap W_{i}\right)$, and $J_{i}$ will be the coefficient ideal in $W_{i}$ of some ideal $K_{i+1}$ associated to $J_{i+1}$ (the ideal $K_{i}$ is essentially the product $I_{i} \cdot Q_{i}$ with $Q_{i}=I_{W_{i}}\left(E_{i} \cap W_{i}\right)$ given by the transversal handicap). The ideals $I_{i}$ and $Q_{i}$ depend on choices, but their orders, which form the components of the invariant $i_{a}(J)$, do not. Thus $i_{a}(J)$ is intrinsic, and upper semicontinuous because orders of ideals are. 
It then has to be shown that the top locus of $i_{a}(J)$ is in fact regular and transversal to the exceptional locus. This allows to choose it as the center of the next blowup. It remains to prove that the transform of the mobile in $W^{\prime}$ admits at each point a setup which is the transform of the setup of the initial mobile (commutativity), thus its invariant can be computed from the invariant below and must have decreased. As the invariant $i_{a}(J)$ can only drop finitely many times, it must eventually achieve its minimal value 0 . In this case, $\operatorname{ord}_{a} I=0$ and $J=M$ as required.

The above proof is based on a cartesian scheme of induction: the descending horizontal induction on the dimension is combined with the vertical induction on the resolution invariant. In total, fourteen inductions are required. Once the relevant objects like mobiles, setups and their transforms are defined properly, the inductions follow always the same pattern.

\section{Concepts}

Throughout, we fix a regular ambient scheme $W$ and a regular locally closed $n$ dimensional subscheme $V$ of $W$. By a divisor in $W$ we shall mean an effective Weil divisor $D$. A closed subscheme $D$ of $W$ has normal crossings if it can be defined locally by a monomial ideal. The subscheme $V$ meets $D$ transversally if the product of the defining ideals of $V$ and $D$ defines a normal crossings scheme.

A local flag in $V$ at $a$ is a decreasing sequence $W_{n} \supset \ldots \supset W_{1}$ of closed $i$ dimensional regular subschemes $W_{i}$ of a neighborhood $U$ of $a$ in $V$. An ideal $K$ in $V$ is bold regular if it is a power of a regular principal ideal in $V$. A stratified ideal in $V$ is a collection of coherent ideal sheaves each of them defined on a stratum of a stratification of $V$ by locally closed subschemes. A stratified divisor is defined by a stratified principal ideal. All ideals and divisors will be stratified without notice, except if said to be coherent. For normal crossings divisors $D$, the underlying stratification strat $(D)$ need not have normal crossings strata.

A map $Q_{b} \rightarrow\left(Q_{b}\right)^{\sharp}$ associating to stalks of ideals $Q_{b}$ in an open subscheme $U$ of $W$ stalks of ideals $\left(Q_{b}\right)^{\sharp}$ in $V$ is tuned along the stratum $S$ of a stratification of $V$ through a point $b$ of $V$ if $Q_{b}$ and $\left(Q_{b}\right)^{\sharp}$ admit locally at any point $b$ of $V$ coherent representatives $\overline{Q_{b}}$ on $U$ and $\overline{\left(Q_{b}\right)^{\sharp}}$ on $V$ so that the stalks $\left(\left(\overline{Q_{b}}\right)_{a}\right)^{\sharp}$ and $\left(\overline{\left(Q_{b}\right)^{\sharp}}\right)_{a}$ at $a$ coincide for $a$ in $S$. This is abbreviated by saying that the ideals $\left(Q_{b}\right)^{\sharp}$ are tuned along the stratum $S$.

A shortcut of a normal crossings divisor $M$ in $W$ is a divisor $N$ obtained from $M$ by deleting on each stratum of the underlying stratification $\operatorname{strat}(M)$ of $M$ some components of $M$. The divisor $M$ is labelled if each shortcut $N$ comes with a different non negative integer lab $N$, its label. The empty shortcut has label 0 . A shortcut $N$ of a normal crossings divisor $M$ is tight at a of order $\geq c$ if it has order $\geq c$ at $a$ and if any proper shortcut of $N$ has order $<c$ at $a$. It is maximal tight at $a$ if $M$ is labelled and if $\left(\operatorname{ord}_{a} N\right.$, lab $\left.N\right)$ is lexicographically maximal among the tight shortcuts of $M$ of order $\geq c$ at $a^{<1>}$.

A handicap on $W$ is a sequence $D=\left(D_{n}, \ldots, D_{1}\right)$ of stratified normal crossings 
divisors $D_{i}$ of $W$. The truncation of $D$ at index $i$ is ${ }^{i} D=\left(D_{n}, \ldots, D_{i}\right)$.

A singular mobile in $W$ is a quadruple $\mathcal{M}=(\mathcal{J}, c, D, E)$ with $\mathcal{J}$ a coherent nowhere zero ideal sheaf on $V, c$ a non negative constant associated to $V$, and $D$ and $E$ handicaps in $W$ with $D$ labelled and $E$ reduced $^{<2>}$. We call $c$ the control of $\mathcal{J}$, and $D$ and $E$ the combinatorial and transversal handicap of $\mathcal{M}$. The truncation ${ }^{i} \mathcal{M}$ at index $i$ of $\mathcal{M}$ is $\left(\mathcal{J}, c,{ }^{i} D,{ }^{i} E\right)$.

The transversality locus of a mobile $\mathcal{M}$ is $|E|=E_{n} \cup \ldots \cup E_{1}$. The exceptional locus of a sequence of blowups of $\mathcal{M}$ is the reduced inverse image of the union of all centers and of $|E|$.

The control $c$ is allowed to be 0 if and only if $\mathcal{J}=1$. Throughout, we denote by calligraphic letters stratified ideal sheaves, and by roman letters their stalks or sufficiently small representatives of them.

A strong resolution of a mobile $\mathcal{M}=(\mathcal{J}, c, D, E)$ in $W$ is a sequence of blowups of $W$ in regular closed centers $Z$ such that the ideal $\mathcal{J}^{\prime}$ of the final transform $\mathcal{M}^{\prime}=\left(\mathcal{J}^{\prime}, c^{\prime}, D^{\prime}, E^{\prime}\right)$ of $\mathcal{M}$ as defined in the section "Transform of mobile" has order $<c$. We require that the centers are transversal to the exceptional loci, and that the resolution is equivariant.

The top locus of an upper semicontinuous function $t$ on $V$ is the reduced closed subscheme top $(t)$ of points of $V$ where $t$ attains its maximum. The order at $a$ of an ideal $\mathcal{J}$ of $V$ is the largest power $o=\operatorname{ord}_{a} \mathcal{J}$ of the maximal ideal of $\mathcal{O}_{V, a}$ containing the stalk $J$ of $\mathcal{J}$ at $a$. We set $\operatorname{top}(\mathcal{J})=\operatorname{top}(\operatorname{ord} \mathcal{J})$ and denote by $\operatorname{top}(\mathcal{J}, c)$ the locus of points in $V$ where the order of $\mathcal{J}$ is at least $c$. For closed subschemes of $V$, the analogous loci are defined through the associated ideals. When working locally at a point $a, \operatorname{top}(t)$ also denotes the local top locus of $t$ in a neighborhood of $a$.

Let $W^{\prime} \rightarrow W$ be the blowup of $W$ with center $Z$ inside $V$ and exceptional component $Y^{\prime}$. The total and weak transform of an ideal $\mathcal{J}$ of $V$ are the inverse image $\mathcal{J}^{*}$ of $\mathcal{J}$ under the induced blowup $V^{\prime} \rightarrow V$ and the ideal $\mathcal{J}^{\curlyvee}=\mathcal{J}^{*} \cdot \mathcal{I}\left(Y^{\prime} \cap\right.$ $\left.V^{\prime}\right)^{-o}$ with $o=\operatorname{ord}_{Z} \mathcal{J}$. The controlled transform of $\mathcal{J}$ with respect to $c \leq o$ is the ideal $\mathcal{J}^{!}=\mathcal{J}^{*} \cdot \mathcal{I}\left(Y^{\prime} \cap V^{\prime}\right)^{-c}$ in $V^{\prime<3>}$.

The companion ideal $P$ of a product $J=M \cdot I$ of ideals in $V$ at $a$ with respect to a control $c \leq \operatorname{ord}_{a} J$ on $V$ is the ideal $P$ in $V$ at $a$ given by

$$
\begin{array}{ll}
P=I+M^{\frac{o}{c-o}} & \text { if } 0<o=\operatorname{ord}_{a} I<c, \\
P=I & \text { otherwise. }
\end{array}
$$

To avoid rational powers of ideals, one could take $P=I^{c-o}+M^{o}$ if $0<o<c$, and $P=I$ otherwise.

Companion ideals are tuned along top $(I) \cap \operatorname{strat}(M)$ and satisfy $\operatorname{top}(P)=\operatorname{top}(I) \cap$ $\operatorname{top}(M, c-o) \subset \operatorname{top}(J, c)$. The transversality ideal $Q$ in $V$ of a normal crossings divisor $E$ of $W$ is the ideal

$$
Q=I_{V}(E \cap V)
$$


defining $E \cap V$ in $V$. The composition ideal $K$ in $V$ of a product $J=M \cdot I$ of ideals in $V$ at $a$ with respect to a control $c$ and a normal crossings divisor $E$ in $W$ equals $<4>$

$$
\begin{array}{ll}
K=P \cdot Q & \text { if } I \neq 1, \\
K=1 & \text { if } I=1,
\end{array}
$$

with $P$ the companion ideal of $J$ and $c$, and $Q$ the transversality ideal of $E$ in $V$. Composition ideals are tuned along $\operatorname{top}(I) \cap \operatorname{strat}(M) \cap \operatorname{strat}(E)$ and satisfy locally $\operatorname{top}(K)=\operatorname{top}(P) \cap \operatorname{top}(Q)$ if $I \neq 1$ and $Q \neq 0$.

The weight on $M$ in the definition of $P$ is necessary to have $P^{\curlyvee}=\left(I+M^{o / c-o}\right)^{\curlyvee}=$ $I^{\curlyvee}+\left(M^{\prime}\right)^{o^{\prime} / c^{\prime}-o^{\prime}}$ when $c^{\prime}=c$ and $o^{\prime}=o$. This ensures that the passage to companion ideals commutes with blowups. The equality $\operatorname{top}(P)=\operatorname{top}(I) \cap \operatorname{top}(M, c-o)$ uses that $\operatorname{ord}_{a} M^{o / c-o}$ is $\geq o$ for $a$ in top $(J)$ since there $c \leq \operatorname{ord}_{a} J=o+\operatorname{ord}_{a} M$. Note that $\operatorname{ord}_{a} P=\operatorname{ord}_{a} I=o$ along $\operatorname{top}(P) \subset \operatorname{top}(I)$, and thus $P=1$ if and only if $I=1$.

In the application, $V$ will be a member $W_{i}$ of the local flag and $E$ will be a member $E_{i}$ of the transversal handicap; the latter does not contain $W_{i}$ and thus $Q_{i} \neq 0$. Moreover, $W_{i}$ will be transversal to $E_{i}$ and thus $\operatorname{ord}_{a} Q_{i}=\operatorname{ord}_{a} E_{i}$, i.e., equal the number of components of $E_{i}$ passing through $a$, since $E_{i}$ is reduced. We have $\operatorname{top}\left(Q_{i}\right)=W_{i} \cap \operatorname{top}\left(E_{i}\right)$ locally at $a$. Therefore any $Z \subset \operatorname{top}\left(Q_{i}\right)$ is contained in the components of $E_{i}$ it meets.

The tag of an ideal $J$ in $V$ at $a$ with control $c$ and normal crossings divisors $D$ and $E$ in $W$ such that $J=M \cdot I$ for $M=I_{V}(D \cap V)$ with $D$ labelled and transversal to $V$ is the vector

$$
t_{a}(J)=(o, k, m) \in \mathbb{N}^{4},
$$

equipped with the lexicographic order. Here, $o=\operatorname{ord}_{a} I$ and $k=\operatorname{ord}_{a} K$ with $K=P \cdot Q$ the composition ideal of $(J, c, E, D)$. We set $m=(0,0)$ if $o>0$, and $m=\left(\operatorname{ord}_{a} N, \operatorname{lab} N\right)$ otherwise with $N$ the maximal tight shortcut of $M$ at $a$ of order $\geq c$.

The combinatorial tag $m$ measures the improvement of the controlled transform of $M$ once we have $J=M$, the singular tag $(o, k)$ measures the improvement of the weak transforms of $I$ and $K$.

The coefficient ideal $<5>$ of an ideal $K$ of $W$ at $a$ with respect to $V$ is an ideal in $V$ which is built from the coefficients of the Taylor expansion of the elements of $K$ with respect to the equations defining $V$. Let $x, y$ and $y$ be regular systems of parameters of $\mathcal{O}_{W, a}$ and $\mathcal{O}_{V, a}$ so that $x=0$ defines $V$ in $W$. For $f$ in $K$ denote by $a_{f, \alpha}$ the elements of $\mathcal{O}_{V, a}$ so that $f=\sum_{\alpha} a_{f, \alpha} \cdot x^{\alpha}$ holds after passage to the completion. Then set

$$
\operatorname{coeff}_{V} K=\sum_{|\alpha|<c}\left(a_{f, \alpha}, f \in K\right)^{\frac{c}{c-|\alpha|}} .
$$

Replacing the exponents by $\frac{c !}{c-|\alpha|}$, rational powers of ideals could be avoided. Coefficient ideals are tuned along $\operatorname{top}(K) \cap V$. Let $V^{\prime} \rightarrow V$ be the blowup of 
$V$ induced by $W^{\prime} \rightarrow W$ with center $Z$ contained in top $(K) \cap V$ and exceptional component $Y^{\prime} \cap V^{\prime}$. At points $a^{\prime}$ of $Y^{\prime} \cap V^{\prime}$ where $c^{\prime}=\operatorname{ord}_{a^{\prime}} K^{\curlyvee}=\operatorname{ord}_{a} K=c$ one has $\left(\operatorname{coeff}_{V} K\right)^{!}=\operatorname{coeff}_{V^{\prime}}\left(K^{\curlyvee}\right)$. Thus the descent to the coefficient ideal commutes with taking the weak, respectively controlled transform at these points.

The proof of commutativity goes as follows, using that $a_{f^{\curlyvee}, \alpha}=\left(a_{f, \alpha}\right)^{*} \cdot I\left(Y^{\prime} \cap V^{\prime}\right)^{|\alpha|-c}$ :

$$
\begin{aligned}
\operatorname{coeff}_{V^{\prime}}\left(K^{\prime}\right) & =\operatorname{coeff}_{V^{\curlyvee}}\left(K^{\curlyvee}\right)= \\
& =\operatorname{coeff}_{V^{\curlyvee}}\left(\sum_{|\alpha|<c^{\prime}} a_{f^{\curlyvee}, \alpha} \cdot x^{\alpha}, f^{\curlyvee} \in K^{\curlyvee}\right)= \\
& =\operatorname{coeff}_{V^{\curlyvee}}\left(\sum_{|\alpha|<c^{\prime}} a_{I\left(Y^{\prime} \cap V^{\prime}\right)^{-c} \cdot f^{*}, \alpha} \cdot x^{\alpha}, f^{\curlyvee} \in K^{\curlyvee}\right)= \\
& =\operatorname{coeff}_{V^{\curlyvee}}\left(\sum_{|\alpha|<c}\left(a_{f, \alpha} \cdot x^{\alpha}\right)^{*} \cdot I\left(Y^{\prime} \cap V^{\prime}\right)^{-c}, f \in K\right)= \\
& =\sum_{|\alpha|<c}\left(a_{f, \alpha}^{*}, f \in K\right)^{c /(c-|\alpha|)} \cdot I\left(Y^{\prime} \cap V^{\prime}\right)^{-c}= \\
& =\left(\sum_{|\alpha|<c}\left(a_{f, \alpha}, f \in K\right)^{c /(c-|\alpha|)}\right)^{*} \cdot I\left(Y^{\prime} \cap V^{\prime}\right)^{-c}= \\
& =I\left(Y^{\prime} \cap V^{\prime}\right)^{-c} \cdot\left(\operatorname{coeff}_{V} K\right)^{*}=\left(\operatorname{coeff}_{V} K\right)^{!} .
\end{aligned}
$$

The coefficient ideal depends on the choice of $V$ and the regular systems of parameters. It satisfies $\operatorname{ord}_{a} \operatorname{coeff}_{V} K \geq \operatorname{ord}_{a} K$ for $a \in V$.

The junior ideal $J$ in $V$ of an ideal $K$ of $W$ at $a$ is the coefficient ideal $\operatorname{coeff}_{V} K$ of $K$ in $V$ if $K$ is not bold regular or 1, and is set equal to 1 otherwise.

The scheme $V$ has weak maximal contact with an ideal $P$ of $W$ at $a$ if $V$ maximizes the order of $\operatorname{coeff}_{V} P$ at $a$. It is osculating $<6>$ for $P$ if there is an $f \in P$ with $\operatorname{ord}_{a} f=\operatorname{ord}_{a} P$ and $\operatorname{ord}_{a} \operatorname{coeff}_{V} f=\operatorname{ord}_{a} \operatorname{coeff}_{V} P$ such that $a_{f, \alpha}=0$ for all $\alpha$ with $|\alpha|=\operatorname{ord}_{a} P-1$.

The coefficient ideal of $K \neq 0$ in a hypersurface of weak maximal contact $V$ is zero if and only if $K$ is bold regular or equal 1 . Namely, if $K$ is bold regular then $\operatorname{top}(K)=V$, by weak maximal contact, and the coefficient ideal is zero. Conversely, if $K \neq 1$ and the coefficient ideal is zero, then $a_{f, \alpha}=0$ for $f \in K$ and $|\alpha|<c$. Thus $K \subset\left(x^{c}\right)$ with $x$ defining $V$ in $W$. Hence the support of $K$ contains the hypersurface defined by $x^{c}$. As the order of $K$ is at most $c$ we get $K=\left(x^{c}\right)$ bold regular.

Junior ideals avoid getting zero ideals $J$ and $I$ having infinite order. They are tuned along $\operatorname{top}(K) \cap V$. The passage to junior ideals in hypersurfaces of weak maximal contact commutes with taking the weak, respectively controlled transform, at points $a^{\prime}$ above $a$ where the order of $K$ has remained constant. This holds for coefficient ideals. If $K$ is not bold regular or 1 but $K^{\prime}$ is this implies that the order of $K$ has dropped, by the commutation of coefficient ideals with weak and controlled transforms. If $K$ is bold regular but not 1 commutativity does not hold except if the center equals the support of $K$. But by construction of $Z$ in the actual resolution process, it does equal the support of $K$ in this case. 
The following properties of osculating hypersurfaces will be used later (see below for proofs.) In characteristic zero, any $a$ in $W$ admits a neighborhood $U$ and a hypersurface $V$ of $U$ which is osculating for $P$ at all points of $\operatorname{top}(P)$ in $U$. If $P \neq 0,1$ then $V$ contains $\operatorname{top}(P) \cap U$ and satisfies $\operatorname{top}\left(J_{P}, c_{P}\right)=\operatorname{top}(P)$ for $c_{P}=\operatorname{ord}_{a} P$ and $J_{P}$ the coefficient ideal of $P$ in $V$. Any $V$ which is osculating for $P$ has weak maximal contact with $P$. If $V$ has weak maximal contact with $P \neq 1$ then it has also weak maximal contact with any product $K=P \cdot Q$.

If $V$ has weak maximal contact with $P$ its weak transform $V^{\curlyvee}$ under blowup of $W$ in a regular center $Z \subset \operatorname{top}(P)$ contains all points $a^{\prime}$ of $W^{\prime}$ where the order of $P^{\curlyvee}$ has remained constant. If $V$ is osculating for $P$, then $V^{\curlyvee}$ is osculating for $P^{\curlyvee}$ at these points. Hence, if $V$ has weak maximal contact with $K=P \cdot Q$ for being osculating for $P$ and if $Z \subset \operatorname{top}(K)$ then $V^{\curlyvee}$ has weak maximal contact with $K^{\curlyvee}=P^{\curlyvee} \cdot Q^{\curlyvee}$ at all points $a^{\prime}$ of $W^{\prime}$ where $\operatorname{ord}_{a^{\prime}} P^{\curlyvee}=\operatorname{ord}_{a} P$, regardless of the order of $K^{\curlyvee}$ at $a^{\prime}$.

We indicate how to prove the more delicate of the above assertions. For the existence of osculating hypersurfaces, take an element $f \in P$ of minimal order $\operatorname{ord}_{a} f=\operatorname{ord}_{a} P$ at $a$. In characteristic 0 , a suitable partial derivative of $f$ of order $\operatorname{ord}_{a} f-1$ has order 1 at $a$ and can be taken as defining equation for $V$. From this, $\operatorname{top}\left(J_{P}, c_{P}\right)=\operatorname{top}(P) \subset V$ is immediate. Equivalently, $V$ can be obtained by a Tschirnhaus coordinate transformation $<6>$.

That osculating with $P$ implies weak maximal contact with $P$ is a direct check on Newton polyhedra. The properties with respect to blowup are classical and proven by computing the transforms of the ideals in local coordinates for which the blowup reduces to a monomial substitution of variables. We show here that weak maximal contact with $P \neq 0,1$ implies weak maximal contact with $K=P \cdot Q$. Let $c, c_{P}$ and $c_{Q}$ be the orders of $K, P$ and $Q$. We may assume that $Q \neq 0,1$, so that all orders are positive and finite. Let $J, J_{P}$ and $J_{Q}$ be the associated coefficient ideals, and $e, e_{P}$ and $e_{Q}$ their respective orders in $\mathbb{N} \cup\{\infty\}$. We claim that

$$
e / c=\min \left\{e_{P} / c_{P}, e_{Q} / c_{Q}\right\}
$$

The equality also holds when $P$ is bold regular, in which case $J_{P}=0$ and $e_{P}=\infty$.

To prove the claim, we fix local coordinates $\left(x, y_{n-1}, \ldots, y_{1}\right)$ at $a$ in $W$ with $V$ defined in $W$ by $x=0$. We treat first the case of single elements $f \in P$ and $g \in Q$. Their Newton polyhedra satisfy $N(f \cdot g)=N(f)+N(g)$, as is shown by a computation of the vertices of $N(f \cdot g)$. Interpret then the order $e_{f \cdot g}$ of the ideal generated by the equilibrated powers of the coefficients of $f \cdot g$ in $J$ as the order of the projected Newton polyhedron $\pi(N(f \cdot g))$ under the projection $\pi$ from the point $(c, 0, \ldots, 0)$ of $\mathbb{N}^{n}=\mathbb{N} \times \mathbb{N}^{n-1}$ to $\mathbb{Q}^{n-1}$ defined for points $(j, \gamma)$ with $j<c$ by $(j, \gamma) \rightarrow \frac{c}{c-j} \cdot \gamma$. (Read $(j, \gamma) \rightarrow \frac{c !}{c-j}$ if you have taken exponent $\frac{c !}{c-|\alpha|}$ in the definition of coefficient ideals and wish to project to $\mathbb{N}^{n-1}$.)

Let $e_{f}$ and $e_{g}$ denote the orders of the ideals generated by the equilibrated powers of the coefficients of $f$ and $g$ in $J_{P}$ and $J_{Q}$ respectively (not of the coefficient ideals of the ideals generated by $f$ and $g$ ). The equality $e_{f \cdot g} / c=\min \left\{e_{f} / c_{P}, e_{g} / c_{Q}\right\}$ then follows from $N(f \cdot g)=$ $N(f)+N(g)$ by a computation in $\mathbb{N}^{n}$.

Write now elements $h \in K$ as $h=\sum_{j} a_{h, j}(y) \cdot x^{j}$ so that $J=\sum_{j<c}\left(a_{h, j}, h \in K\right)^{c / c-j}$, and similarly for $P$ and $Q$. There exists a finite generator set $H$ of $K$ such that $J=$ $\sum_{j<c}\left(a_{h, j}, h \in H\right)^{c / c-j}$. Let $F$ and $G$ be defined analogously for $P$ and $Q$. Each $h \in H$ is a linear combination of products $f \cdot g$ with $f \in F$ and $g \in G$. Enlarging $F$ and $G$ we may assume that all coefficients in the sum are 0 or 1 . Replacing $H$ by all summands of all $h$ we 
obtain $H=F \cdot G$. The coefficients of the elements of the new $H$ generate again $J$. By the formula above we get

$$
\begin{aligned}
e / c & =\min _{h \in H} e_{h} / c= \\
& =\min _{f g \in H} \min \left\{e_{f} / c_{P}, e_{g} / c_{Q}\right\}= \\
& =\min \left\{\min _{f \in F} e_{f} / c_{P}, \min _{g \in G} e_{g} / c_{Q}\right\}= \\
& =\min \left\{e_{P} / c_{P}, e_{Q} / c_{Q}\right\} .
\end{aligned}
$$

This proves the claim. Assume now that $V$ has weak maximal contact with $P$, i.e., that $e_{P}$ is maximal. If $e_{P} / c_{P} \leq e_{Q} / c_{Q}$ then $e / c$ and hence $e$ is already maximal, by the above formula. So assume that $e_{P} / c_{P}>e_{Q} / c_{Q}$, and that $e / c=e_{Q} / c_{Q}$ is not maximal. Increasing $e_{Q} / c_{Q}$ by a coordinate change requires up to permutations a change $(x, y) \rightarrow(x+g(y), y)$ with slope ord $g=e_{Q} / c_{Q}$.

Let $P^{\sim}$ and $Q^{\sim}$ be the resulting ideals. Then, as $P \neq 0,1$ and $e_{P} / c_{P}>e_{Q} / c_{Q}$, we get $e_{P} \sim / c_{P} \sim=e_{Q} / c_{Q}$, hence $e / c=\min \left\{e_{P} \sim / c_{P} \sim, e_{Q} \sim / c_{Q} \sim\right\}=e_{Q} / c_{Q}$ remains constant, i.e., was already maximal. This shows that weak maximal contact with $P \neq 0,1$ implies weak maximal contact with $K=P \cdot Q$.

\section{Setup}

Let $\mathcal{M}=(\mathcal{J}, c, D, E)$ be a singular mobile in $W$ with $\mathcal{J}$ a coherent ideal in a locally closed regular $n$-dimensional subscheme $V$. Write $J_{n}$ for the stalk of $\mathcal{J}$ at a point $a$ of $V$. A punctual setup $<7>$ of $\mathcal{M}$ at $a$ is a sequence $\left(J_{n}, \ldots, J_{1}\right)$ of stalks of ideals $J_{i}$ in a local flag $\left(W_{n}, \ldots, W_{1}\right)$ of $V$ at $a$ satisfying for all $i \leq n$

(1) $J_{i}=M_{i} \cdot I_{i}$ with $M_{i}=I_{W_{i}}\left(D_{i} \cap W_{i}\right)$ and $I_{i}$ an ideal in $W_{i}$ at $a$.

(2) $M_{i}$ defines a normal crossings divisor in $W_{i}$ at $a$.

(3) $W_{i-1}$ has weak maximal contact at $a$ with the composition ideal $K_{i}$ in $W_{i}$ of $\left(J_{i}, c_{i+1}, D_{i}, E_{i}\right)$. Here, $c_{i+1}$ is the control of $J_{i}$ on $W_{i}$. It is given for $i<n$ as the order of $K_{i+1}$ in $W_{i+1}$ at $a$, and $c_{n+1}=c$.

(4) $J_{i-1}$ is the junior ideal of $K_{i}$ in $W_{i-1}$.

Setups depend on and are determined by the choice of the local flag subject to the above conditions. They commute with the operations described in equivariance.

Let $d$ be maximal with $o_{d}=0$, and set $d=0$ if all $o_{i}=\operatorname{ord}_{a} I_{i}>0$. From (3) follows that $K_{i}=P_{i} \cdot Q_{i}$ for $i>d$, with $P_{i}=I_{i}+M_{i}^{o_{i} /\left(c_{i+1}-o_{i}\right)}$ and $Q_{i}=I_{W_{i}}\left(E_{i} \cap W_{i}\right)$. For $i \leq d$ we have $K_{i}=1$.

A tuned setup of $(\mathcal{J}, c, D, E)$ on an open subscheme $U$ of $V$ is a sequence of coherent ideal sheaves $\mathcal{J}_{n}, \ldots, \mathcal{J}_{1}$ in a decreasing flag $W_{n}, \ldots, W_{1}$ of closed subschemes of $U$ such that, for any $i \leq n$, the stalks $J_{n}, \ldots, J_{i}$ at $a$ define the truncation of a punctual setup of $(\mathcal{J}, c, D, E)$ for all points $a$ of $U \cap \operatorname{top}\left(t_{n}, \ldots, t_{i+1}\right)$. Here, $t_{i}=\left(o_{i}, k_{i}, m_{i}\right)$ denotes the tag of $\left(J_{i}, c_{i+1}, D_{i}, E_{i}\right)$ at $a$.

Thus $o_{i}=\operatorname{ord}_{a} I_{i}, k_{i}=\operatorname{ord}_{a} K_{i}$ in $W_{i}$, and $m_{i}$ is the combinatorial tag of the maximal tight shortcut $N_{i}$ of $M_{i}$ of order $\geq c_{i+1}$ at $a$. The restriction $\left(J_{i-1}, \ldots, J_{1}\right)$ is a punctual 
setup at $a$ in $W_{i-1}$ of the restricted mobile $\left(J_{i-1}, c_{i}, D^{i-1}, E^{i-1}\right)$ in $W_{i-1}$ where $D^{i-1}=$ $\left(D_{i-1}, \ldots, D_{1}\right)$ and $E^{i-1}=\left(E_{i-1}, \ldots, E_{1}\right)$.

Indices indicate, except for handicaps, the dimension of the corresponding ambient scheme. The order of an ideal is taken with respect to this ambient scheme. Observe that, by definition, $c_{i+1}$ is constant on $W_{i}$ and only defined there, whereas $D_{i}$ and $E_{i}$ are defined in $W$. More accurately we should write $W_{i}=W_{i}(a)$. Then, locally at a point $b$, we will be able to choose $W_{i}$ so that $W_{i}(a)=W_{i}(b)$ for all $a$ in $\operatorname{top}\left(K_{i+1}\right)$ near $b$.

\section{Invariant}

Let $\mathcal{M}=(\mathcal{J}, c, D, E)$ be a mobile in $W$ with $\mathcal{J}$ a non zero coherent ideal sheaf in $V$. Assume that $\mathcal{M}$ admits locally on $V$ tuned setups with induced punctual setups $\left(J_{n}, \ldots, J_{1}\right)$. Set

$$
i_{a}(\mathcal{M})=\left(t_{n}, \ldots, t_{1}\right) \in \mathbb{N}^{4 n}
$$

with $t_{i}=\left(o_{i}, k_{i}, m_{i}\right)$ the tag of $\left(J_{i}, c_{i+1}, D_{i}, E_{i}\right)$ at $a^{<8>}$. Equipping $\mathbb{N}^{4 n}$ with the lexicographic order this vector satisfies the following properties.

(a) $i_{a}(\mathcal{M})$ does not depend on the chosen setup of $\mathcal{M}$ at $a$ and commutes with the operations described in equivariance.

(b) The map $a \rightarrow i_{a}(\mathcal{M})$ is upper semicontinuous on $V$. The induced stratification of $V$ refines the stratification underlying $D$ and $E$.

(c) The top locus $Z$ of $i_{a}(\mathcal{M})$ is regular. Locally, $Z$ lies in the top loci of all $I_{i}$, $P_{i}, Q_{i}$ and $K_{i}$. It only depends on the restriction of $i_{a}(\mathcal{M})$ to the support of $\mathcal{J}$.

(d) $Z$ is transversal to all $D_{i}$ and $E_{i}$.

The first part of properties (a) and (b) will be proven in the section "Independence and semicontinuity", the second part of (a) holds by construction of $i_{a}(\mathcal{M})$, and the second part of property (b) is proven in "Transform of mobile". The first two parts of property (c) are proven in "Top loci". The third part holds by construction of $i_{a}(\mathcal{M})$, since its first component $o_{n}=\operatorname{ord}_{a} I_{n}$ has top locus inside the support of $\mathcal{J}$ and all remaining components defining $Z$ are orders of ideals taken at points of this locus. Property (d) is proven in "Transversality".

Equivariance of the invariant signifies that the invariant remains the same under smooth morphisms and field extensions. And, if $W \rightarrow W^{+}$is a closed embedding of $W$ into a regular scheme $W^{+}$, and $\mathcal{M}^{+}$a mobile in $W^{+}$inducing by restriction to $W$ the mobile $\mathcal{M}$, then for $a$ in $W$ the restriction of $i_{a}\left(\mathcal{M}^{+}\right)$to dimension $n=\operatorname{dim} W$ equals the invariant $i_{a}(\mathcal{M})$. We will use this property in "Resolution of schemes" only in case the divisors of the handicaps $D$ and $E$ of $\mathcal{M}$ are empty with the exception of $E_{n} \subset W$.

The first two properties of equivariance hold because they hold for order of ideals and because mobiles and their setups commute with these operations. The third property follows from the fact that the order of the ideal $J^{+}$in $W^{+}$extending $J$ is 1 if $\operatorname{dim} W<\operatorname{dim} W^{+}$. If $D_{n^{+}}^{+}$and $E_{n^{+}}^{+}$are empty and $\operatorname{dim} W<\operatorname{dim} W^{+}$, the first composition ideal $K^{+}$equals $J^{+}$, and its coefficient ideal in a hypersurface $V^{+}$of $W^{+}$is just the restriction of $J^{+}$to $V^{+}$. 
This hypersurface can be chosen to contain $W$. By induction on the dimension, we reach by iteration a coefficient ideal which equals $J$, and the first components of $i_{a}\left(\mathcal{M}^{+}\right)$are either 1 (as for $o$ and $k$ ) or 0 (as for $m$ ). Therefore the invariant of $\mathcal{M}^{+}$restricts on $W$ to the invariant of $\mathcal{M}$. We omit the reasoning for arbitrary $D^{+}$and $E^{+}$.

\section{Comments}

Mobiles are the minimal resolution datum needed to define at each stage of the resolution process the local invariant. They present global information and only depend on the initial mobile and the transformation laws: $\mathcal{J}$ is the ideal we wish to resolve, i.e., to transform into a principal monomial ideal supported by exceptional components. The control $c$ indicates how $\mathcal{J}$ transforms under blowup; in addition, it prescribes the objective: to drop the order of the transforms of $\mathcal{J}$ below $c$. The components $D_{i}$ of $D$ keep track which part of $J_{i}$ has already been monomialized. The components $E_{i}$ of $E$ collect the exceptional components which may fail to be transversal to $W_{i-1}$. They both live in $W$ and are independent of the choice of the local flags, whereas the associated ideals $M_{i}=I_{W_{i}}\left(D_{i} \cap W_{i}\right)$ and $Q_{i}=I_{W_{i}}\left(E_{i} \cap W_{i}\right)$ live in $W_{i}$ and depend on the flag.

Setups are auxiliary local data depending on the flags. They are defined so that the resulting invariant does not depend on the chosen flag. Their tunedness ensures the semicontinuity of the invariant. The passage from $I_{i}$ to $P_{i}$ is necessary because $\operatorname{top}\left(I_{i}\right)$ need not be contained in $\operatorname{top}\left(J_{i}, c_{i+1}\right)$ when the order of $I_{i}$ has become small. Multiplication of $P_{i}$ with $Q_{i}$ allows to treat the transversality problem with the exceptional components simultaneously with the monomialization of $J_{i}$. The inclusions

$$
\operatorname{top}\left(I_{i}\right) \supset \operatorname{top}\left(P_{i}\right), \quad \operatorname{top}\left(E_{i}\right)=\operatorname{top}\left(Q_{i}\right) \supset \operatorname{top}\left(K_{i}\right)
$$

and

$$
\ldots \supset \operatorname{top}\left(K_{i+1}\right) \supset \operatorname{top}\left(J_{i}, c_{i+1}\right) \supset \operatorname{top}\left(P_{i}\right) \supset \operatorname{top}\left(K_{i}\right) \supset \ldots \supset Z
$$

imply that the orders of the weak transforms of $I_{i}$ and $K_{i}$ do not increase under blowup. It is shown that they can only remain constant in a very specific situation, in which case the combinatorial tag $m_{i}$ drops. This establishes the vertical induction on the resolution invariant.

Weak maximal contact may not persist under blowup, and osculating hypersurfaces are a characteristic zero device to achieve this persistence. For technical reasons it is appropriate to take $W_{i}$ osculating with $P_{i+1}$ instead of $K_{i+1}=$ $P_{i+1} \cdot Q_{i+1}$. The key point of the proof is the commutativity of the descent in dimension via coefficient ideals with the passage to points of the blowup where the order has remained constant. This allows descending induction on the dimension. 


\section{Transform of mobile}

Suppose given a mobile with empty combinatorial handicap. By horizontal induction on the dimension it admits locally tuned setups. Thus the invariant is defined. Its top locus is closed and regular and gives the center of the first blowup.

Assume then constructed at a certain stage of the resolution process the mobile $\mathcal{M}=(\mathcal{J}, c, D, E)$ in $W$ with $\mathcal{J}$ an ideal of control $c$ in $V$ and locally tuned setups with invariant $i(\mathcal{M})$. If $\mathcal{J}$ has order $<c$ on $V$ the mobile is resolved. If not, let $W^{\prime} \rightarrow W$ be the blowup of $W$ in the top locus $Z$ of $i(\mathcal{M})$ with new exceptional component $Y^{\prime}$. The transform $\mathcal{M}^{\prime}=\left(\mathcal{J}^{\prime}, c^{\prime}, D^{\prime}, E^{\prime}\right)$ of $\mathcal{M}$ and a locally defined tuned setup of $\mathcal{M}^{\prime}$ are constructed as follows $<9>$.

By the first component of $i_{a}(\mathcal{M})$ we will know that the center $Z$ is contained in $V$ thus inducing a blowup $V^{\prime} \rightarrow V$. If the controlled transform $\mathcal{J}^{!}$of $\mathcal{J}$ in $V^{\prime}$ has order $<c$ on $V^{\prime}$ the resolution of $\mathcal{J}$ is completed. If not, set $\mathcal{J}^{\prime}=\mathcal{J}^{\text {! }}$ and $c^{\prime}=c$. Let $a^{\prime}$ be a point of $V^{\prime}$ and denote by $J_{n}^{\prime}$ the stalk of $\mathcal{J}^{\prime}$ at $a^{\prime}$. Assume constructed for some $i<n$ the truncated mobile ${ }^{i+1} \mathcal{M}^{\prime}$, and, locally on $V^{\prime}$, flags $W_{n}^{\prime}, \ldots, W_{i+1}^{\prime}$ with truncated tuned $\operatorname{setup} \mathcal{J}_{n}^{\prime}, \ldots, \mathcal{J}_{i+1}^{\prime}$ of ${ }^{i+1} \mathcal{M}^{\prime}$.

We shall define the truncated mobile ${ }^{i} \mathcal{M}^{\prime}$ and, locally on $W_{i+1}^{\prime}$, regular hypersurfaces $W_{i}^{\prime}$, such that the junior ideal $J_{i}^{\prime}$ of $K_{i+1}^{\prime}$ in $W_{i}^{\prime}$ yields a punctual truncated setup $J_{n}^{\prime}, \ldots, J_{i}^{\prime}$ of ${ }^{i} \mathcal{M}^{\prime}$ induced from a tuned truncated setup $\mathcal{J}_{n}^{\prime}, \ldots, \mathcal{J}_{i}^{\prime}$. The transform $\mathcal{M}^{\prime}$ of $\mathcal{M}$ is then defined by descending induction on $i$ and will admit locally tuned setups.

For $a^{\prime} \in W^{\prime}$, let $J_{n}^{\prime}, \ldots, J_{i+1}^{\prime}$ denote the truncated punctual setup of ${ }^{i+1} \mathcal{M}^{\prime}$ induced by $\mathcal{J}_{n}^{\prime}, \ldots, \mathcal{J}_{i+1}^{\prime}$. Thus $\left(t_{n}^{\prime}, \ldots, t_{i+1}^{\prime}\right)$ is defined. By "Independence and semicontinuity" it will be independent of the choice of the flag.

For $n \geq j \geq i+1$, let $T_{j+1}^{\prime}$ be the locus of points in $W^{\prime}$ where $\left(t_{n}^{\prime}, \ldots, t_{j+1}^{\prime}\right)$ equals the value of $\left(t_{n}, \ldots, t_{j+1}\right)$ along $Z$. We agree that $T_{n+1}^{\prime}=W_{n}^{\prime}$. Let $O_{j}^{\prime}$ be the locus in $T_{j+1}^{\prime}$ where $o_{j}^{\prime}$ is positive and equal the value of $o_{j}$ along $Z$. The independence of the truncated invariant implies that these loci do not depend on the chosen flags. By descending horizontal induction on $i$ we shall assume that the handicaps of ${ }^{i+1} \mathcal{M}^{\prime}$ satisfy for all $j \geq i+1$ the equalities

$$
\begin{aligned}
& D_{j}^{\prime}=D_{j}^{*}+\left(o_{j}-c_{j+1}\right) \cdot Y^{\prime} \quad \text { on } T_{j+1}^{\prime} \text {, } \\
& D_{j}^{\prime}=\emptyset \quad \text { outside } T_{j+1}^{\prime} \text {, } \\
& E_{j}^{\prime}=E_{j}^{\curlyvee} \quad \text { on } O_{j}^{\prime}, \\
& E_{j}^{\prime}=\left(Y^{\prime}+|E|^{\curlyvee}\right)-\left(E_{n}^{\prime}+\ldots+E_{j+1}^{\prime}\right) \quad \text { outside } O_{j}^{\prime} .
\end{aligned}
$$

Here, $D_{j}^{*}$ denotes the pull-back of $D_{j}$ under $W^{\prime} \rightarrow W$. Define $T_{i+1}^{\prime}$ and $D_{i}^{\prime}$ by setting $j=i$ in the above formulas. Then $D_{i}^{\prime}$ does not depend on the chosen flags. It is stratified with underlying stratification given by $T_{i+1}^{\prime}$. This establishes the second part of property (b) of the invariant for $D_{i}^{\prime}$. It is shown in "Transversality" that $D_{i}^{\prime}$ is a normal crossings divisor in $W^{\prime}$. It is labelled as follows. Shortcuts which do not involve $Y^{\prime}$ get the label of their image in $W$. The remaining shortcuts are labelled arbitrarily by distinct and pairwise different numbers. 
The coherence of $D_{i}^{\prime}$ along $T_{i+1}^{\prime}$ is proven by vertical induction. Assume that $D_{i}$ is coherent along $T_{i+1}$. If $T_{i+1}^{\prime}=\emptyset$, nothing is to show. If not, $T_{i+1}^{\prime}=\operatorname{top}\left(t_{n}^{\prime}, \ldots, t_{i+1}^{\prime}\right)$ lies over top $\left(t_{n}, \ldots, t_{i+1}\right)$, since the truncated invariant does not increase under blowup, see "Decrease of invariant". This and the coherence of $D_{i}$ on top $\left(t_{n}, \ldots, t_{i+1}\right)$ imply that $D_{i}^{*}$ is coherent on $T_{i+1}^{\prime}$. As both $o_{i}$ and $c_{i+1}$ are constant on $Z$, the factor $I\left(Y^{\prime}\right)^{o_{i}-c_{i+1}}$ is coherent on $Y^{\prime}$ and hence on $W^{\prime}$. Therefore $D_{i}^{\prime}$ is coherent on $T_{i+1}^{\prime}$.

Let $K_{i+1}^{\prime}$ be the composition ideal of $\left(J_{i+1}^{\prime}, c_{i+2}^{\prime}, D_{i+1}^{\prime}, E_{i+1}^{\prime}\right)$ in $W_{i+1}^{\prime}$. It is stratified in $W_{i+1}^{\prime}$ with strata given by the order $o_{i+1}^{\prime}$ of $I_{i+1}^{\prime}$ and by the stratifications underlying $D_{i+1}^{\prime}$ and $E_{i+1}^{\prime}$. Choose in a neighborhood of $a^{\prime}$ a hypersurface $W_{i}^{\prime}$ in $W_{i+1}^{\prime}$ which has weak maximal contact with $K_{i+1}^{\prime}$ along top $\left(K_{i+1}^{\prime}\right)$.

Define $J_{i}^{\prime}$ as the junior ideal of $K_{i+1}^{\prime}$ in $W_{i}^{\prime}$. In "Commutativity" it is shown that $W_{i}^{\prime}$ can be taken osculating for $P_{i+1}^{\prime}$, and that then $J_{n}^{\prime}, \ldots, J_{i}^{\prime}$ form a truncated punctual setup of ${ }^{i} \mathcal{M}^{\prime}$ along $\operatorname{top}\left(t_{n}^{\prime}, \ldots, t_{i+1}^{\prime}\right)$. In particular, $J_{i}^{\prime}=M_{i}^{\prime} \cdot I_{i}^{\prime}$ with $M_{i}^{\prime}=I_{W_{i}^{\prime}}\left(D_{i}^{\prime} \cap W_{i}^{\prime}\right)$ a normal crossings divisor in $W_{i}^{\prime}$, and $o_{i}^{\prime}=\operatorname{ord}_{a^{\prime}} I_{i}^{\prime}$ is defined for $a^{\prime}$ in $W_{i}^{\prime}$. Let $O_{i}^{\prime}$ be the locus of points $a^{\prime}$ in $T_{i+1}^{\prime}$ where $o_{i}^{\prime}$ is positive and equal the value of $o_{i}$ along $Z$. In "Independence and semicontinuity" it is shown that $o_{i}^{\prime}$ and hence $O_{i}^{\prime}$ do not depend on the choice of $W_{i}^{\prime}$. Define

$$
\begin{aligned}
& E_{i}^{\prime}=E_{i}^{\curlyvee} \\
& \text { on } O_{i}^{\prime} \text {, } \\
& E_{i}^{\prime}=\left(Y^{\prime}+|E|^{\curlyvee}\right)-\left(E_{n}^{\prime}+\ldots+E_{i+1}^{\prime}\right) \quad \text { outside } O_{i}^{\prime} .
\end{aligned}
$$

The divisor $E_{i}^{\prime}$ shall collect the exceptional components which may not be transversal to $W_{i-1}^{\prime}$. As $W_{i-1}^{\prime}$ will equal on $O_{i}^{\prime}$ the transform of $W_{i-1}$ the new exceptional component $Y^{\prime}$ will be transversal to $W_{i-1}^{\prime}$, so that we set $E_{i}^{\prime}=E_{i}^{\curlyvee}$ on $O_{i}^{\prime}$. Outside, a new $W_{i-1}^{\prime}$ will be chosen, and hence need not be transversal to any exceptional component. Thus $E_{i}^{\prime}$ consists of all exceptional components except those taken care of in $E_{n}^{\prime}+\ldots+E_{i+1}^{\prime}$. Observe that $Z \subset \operatorname{top}\left(P_{i}\right) \cap \operatorname{top}\left(Q_{i}\right)$ implies that $Z$ is contained for $i>d$ in the components of $E_{i}$ it meets, and is hence transversal to all components of $E_{i}$.

It is shown in "Transversality" that $E_{i}^{\prime}$ is a reduced normal crossings divisor in $W^{\prime}$. It does not depend on the flag $W_{n}^{\prime}, \ldots, W_{i}^{\prime}$. By vertical induction, we may assume that $E_{i}$ is coherent along $O_{j} \backslash O_{j-1}$ for all $j \geq i$. As $O_{j}^{\prime}=\operatorname{top}\left(t_{n}^{\prime}, \ldots, t_{j+1}^{\prime}, o_{j}^{\prime}\right)$ lies over top $\left(t_{n}, \ldots, t_{j+1}, o_{j}\right)$ we conclude that $E_{i}^{\prime}$ is coherent along $O_{j}^{\prime} \backslash O_{j-1}^{\prime}$ for all $j \geq i$, proving for $E_{i}^{\prime}$ the second part of property (b) of the invariant. This completes the construction of ${ }^{i} \mathcal{M}^{\prime}$ and of its locally tuned truncated setups $\mathcal{J}_{n}^{\prime}, \ldots, \mathcal{J}_{i}^{\prime}$. The transform $\mathcal{M}^{\prime}$ of $\mathcal{M}$ and the ideals $\mathcal{J}_{n}^{\prime}, \ldots, \mathcal{J}_{1}^{\prime}$ are then defined by descending horizontal induction on $i$.

\section{Logical structure of proof}

In "Top loci" it is shown by descending horizontal induction on the dimension that, locally at points $a$ in $W$, the center $Z$ is contained in the members $W_{i}$ of the local flag at $a$ for all $i \geq d$, where $d$ is maximal with $o_{d}=0$.

In "Commutativity" it is shown, assuming that the assertions of the later section "Transversality" hold at the present stage $W$ of the resolution process, that at 
points of $W^{\prime}$ where a truncation of the invariant at a certain index $i$ has remained constant, the subsequent descent in dimension and the truncation at the next index $i-1$ commute with blowup. This and the inclusions of "Top loci" allow to show in "Decrease of invariant" that the complete invariant cannot increase when passing from $W$ to $W^{\prime}$. From horizontal induction on the dimension then follows that it actually decreases. This in turn is used together with "Commutativity" and "Top loci" to show in "Transversality" that the handicaps in $W^{\prime}$ are normal crossings divisors and transversal to the next center. In this way, the circle of implications winds up like a spiral through the resolution process. The sections "Independence and semicontinuity" and "Order of coefficient ideals" show that the invariant does not depend on the local choices and is upper semicontinuous.

\section{Top loci}

Let $\left(J_{n}, \ldots, J_{1}\right)$ be a punctual setup of $\mathcal{M}$ at $a$. Let $d$ be maximal with $o_{d}=0$. We show that $\operatorname{top}\left(t_{n}, \ldots, t_{d+1}\right)$ coincides with $\operatorname{top}\left(t_{d+1}\right)=\operatorname{top}\left(o_{d+1}, k_{d+1}\right)=$ $\operatorname{top}\left(K_{d+1}\right)$ in $W_{d+1}$. Locally at $a$ this locus lies in $W_{d}$ if $d \geq 1$, and equals $a$ if $d=0$.

Assume that $\operatorname{top}\left(t_{n}, \ldots, t_{i+1}\right)=\operatorname{top}\left(t_{i+1}\right)=\operatorname{top}\left(K_{i+1}\right)$ holds for some $i>d$. Then $\operatorname{top}\left(t_{n}, \ldots, t_{i+1}, t_{i}\right)=\operatorname{top}\left(t_{i+1}, t_{i}\right)=\operatorname{top}\left(t_{i}\right)$ in $W_{i}$ because $\operatorname{top}\left(t_{i+1}\right)=$ $\operatorname{top}\left(K_{i+1}\right)$ contains $\operatorname{top}\left(J_{i}, c_{i+1}\right) \supset \operatorname{top}\left(P_{i}\right)$ and hence top $\left(t_{i}\right)$. Descending horizontal induction then yields the assertion.

We show that $Z=\operatorname{top}(i(\mathcal{M}))$ equals, locally at $a$, the top locus in $W_{d}$ of the maximal tight shortcut $N_{d}$ of $M_{d}$ of order $\geq c_{d+1}$ if $d \geq 1$, and $Z=\{a\}$ if $d=0$. In the second case, $K_{1}$ is bold regular and different from 1 in $W_{1}$ with support $Z=\{a\}$. If $d \geq 1$ then $o_{d}=0$ implies $K_{d}=1, k_{d}=0$, so that $t_{d}=\left(0,0, m_{d}\right)$ and the remaining components of the invariant are zero. By the above, $Z=\operatorname{top}(i(\mathcal{M}))$ lies in $W_{d}$. It hence equals $\operatorname{top}\left(m_{d}\right)=\operatorname{top}\left(N_{d}\right)$ in $W_{d}$.

\section{Commutativity}

Suppose given a mobile $\mathcal{M}=(\mathcal{J}, c, D, E)$ in $W$ at a certain stage of the resolution process, with truncated transform ${ }^{i+1} \mathcal{M}^{\prime}$ in $W^{\prime}$ as defined in "Transform of mobile", for some $i<n$.

Assume constructed truncated punctual setups $\left(J_{n}, \ldots, J_{i+1}\right)$ of ${ }^{i+1} \mathcal{M}$ in local flags $W_{n}, \ldots, W_{i+1}$ at $a$ and $\left(J_{n}^{\prime}, \ldots, J_{i+1}^{\prime}\right)$ of ${ }^{i+1} \mathcal{M}^{\prime}$ in local flags $W_{n}^{\prime}, \ldots, W_{i+1}^{\prime}$ at $a^{\prime}$ such that $W_{j}^{\prime}=W_{j}^{\curlyvee}$ on $O_{j+1}^{\prime}, J_{j}^{\prime}=J_{j}^{!}$and $I_{j}^{\prime}=I_{j}^{\curlyvee}$ on $T_{j+1}^{\prime}$, and $P_{j}^{\prime}=P_{j}^{\curlyvee}$, $Q_{j}^{\prime}=Q_{j}^{\curlyvee}$ and thus $K_{j}^{\prime}=K_{j}^{\curlyvee}$ on $O_{j}^{\prime}$ for all $j \geq i+1$. In particular, $D_{i}^{\prime}$ can then be defined as in "Transform of mobile". Assume also that $W_{j}$ is chosen osculating for $P_{j+1}$ at $a$ if $j \geq i$, and that $W_{j}^{\prime}$ is chosen osculating for $P_{j+1}^{\prime}$ at $a^{\prime}$ if $j \geq i+1$.

We show that there exists, locally at $a^{\prime}$ in $W_{i+1}^{\prime}$, a regular hypersurface $W_{i}^{\prime}$ which is osculating for $P_{i+1}^{\prime}$ and such that the above commutativity relations also hold for $j=i$, where the ideals $J_{i}^{\prime}, I_{i}^{\prime}, P_{i}^{\prime}, Q_{i}^{\prime}$ and $K_{i}^{\prime}$ are defined as in "Transform of mobile". This allows in particular to define $E_{i}^{\prime}$ and the truncated mobile ${ }^{i} \mathcal{M}^{\prime}$ as 
in "Transform of mobile". Moreover, $\left(J_{n}^{\prime}, \ldots, J_{i}^{\prime}\right)$ will define a truncated punctual setup of ${ }^{i} \mathcal{M}^{\prime}$ at $a^{\prime}$, completing the induction step from $i+1$ to $i$.

For $W_{j}^{\prime}=W_{j}^{\curlyvee}$ we need that $P_{j+1}^{\prime}=P_{j+1}^{\curlyvee}$ and $o_{j+1}^{\prime}=o_{j+1}$ on $O_{j+1}^{\prime}$. For $J_{j}^{\prime}=J_{j}^{!}$we need that $K_{j+1}^{\prime}=K_{j+1}^{\curlyvee}$ and $k_{j+1}^{\prime}=k_{j+1}$ on $T_{j+1}^{\prime}$. For $I_{j}^{\prime}=I_{j}^{\curlyvee}$ we need that $K_{j+1}^{\prime}=K_{j+1}^{\curlyvee}$ and $k_{j+1}^{\prime}=k_{j+1}$ and $D_{j}^{\prime}=D_{j}^{*}+\left(o_{j}-c_{j+1}\right) \cdot Y^{\prime}$ on $T_{j+1}^{\prime}$. For $P_{j}^{\prime}=P_{j}^{\curlyvee}$ we need that $I_{j}^{\prime}=I_{j}^{\curlyvee}$ and $o_{j}^{\prime}=o_{j}$ on $O_{j}^{\prime}$. For $Q_{j}^{\prime}=Q_{j}^{\curlyvee}$ we need that $E_{j}^{\prime}=E_{j}^{\curlyvee}$ on $O_{j}^{\prime}$. For $K_{j}^{\prime}=K_{j}^{\curlyvee}$ we need that $P_{j}^{\prime}=P_{j}^{\curlyvee}$ and $Q_{j}^{\prime}=Q_{j}^{\curlyvee}$ on $O_{j}^{\prime}$ for $j>d^{\prime}=d$, respectively $o_{j}^{\prime}=o_{j}=0$ on $O_{j}^{\prime}$ for $j \leq d^{\prime}$. As $Z \subset \operatorname{top}\left(P_{j}\right) \cap \operatorname{top}\left(Q_{j}\right)$ for $j>d$ and hence $o_{j}=\operatorname{ord}_{a} P_{j}=\operatorname{ord}_{Z} P_{j}$ and $r_{j}=\operatorname{ord}_{a} Q_{j}=$ $\operatorname{ord}_{Z} Q_{j}$ we get on $O_{j}^{\prime}$ from $P_{j}^{\prime}=P_{j}^{\curlyvee}$ and $Q_{j}^{\prime}=Q_{j}^{\curlyvee}$ for $j>d^{\prime}$ that

$$
\begin{aligned}
K_{j}^{\prime} & =P_{j}^{\prime} \cdot Q_{j}^{\prime}=P_{j}^{\curlyvee} \cdot Q_{j}^{\curlyvee}= \\
& =P_{j}^{*} \cdot Q_{j}^{*} \cdot I_{W_{j}^{\prime}}\left(Y^{\prime} \cap W_{j}^{\prime}\right)^{-\left(o_{j}+r_{j}\right)}= \\
& =\left(P_{j} \cdot Q_{j}\right)^{\curlyvee}=K_{j}^{\curlyvee} .
\end{aligned}
$$

Observe that we have $I_{j}^{\prime}=I_{j}^{\curlyvee}$ on $T_{j+1}^{\prime}$, whereas $P_{j}^{\prime}=P_{j}^{\curlyvee}$ holds only on $O_{j}^{\prime}$, by the very definition of companion ideals.

As $W_{i}$ is osculating for $P_{i+1}$ it has weak maximal contact with $P_{i+1}$ and hence with $K_{i+1}$. As $P_{i+1}^{\prime}=P_{i+1}^{\curlyvee}$ holds on $O_{i+1}^{\prime}$ and has order $o_{i+1}^{\prime}=o_{i+1}$ there, $W_{i}^{\gamma}$ is osculating for $P_{i+1}^{\prime}$, hence has weak maximal contact with $K_{i+1}^{\prime}$ on $O_{i+1}^{\prime}$. We set $W_{i}^{\prime}=W_{i}^{\curlyvee}$ on $O_{i+1}^{\prime}$. Outside $O_{i+1}^{\prime}$ we take locally along $\operatorname{top}\left(P_{i+1}^{\prime}\right)$ for $W_{i}^{\prime}$ any hypersurface in $W_{i+1}^{\prime}$ which is osculating for $P_{i+1}^{\prime}$. Let $J_{i}$ and $J_{i}^{\prime}$ be the junior ideals of $K_{i+1}$ and $K_{i+1}^{\prime}$ in $W_{i}$ and $W_{i}^{\prime}$ respectively.

We use here that points where the order of $P_{i+1}^{\prime}$ has remained constant lie in $W_{i}^{\curlyvee}$. Note that the local choices of $W_{i}^{\prime}$ need not patch globally along top $\left(K_{i+1}^{\prime}\right)$.

As the descent to coefficient ideals commutes with weak, respectively controlled transforms, we get by definition of junior ideals that $J_{i}^{\prime}=J_{i}^{!}$on $T_{i+1}^{\prime}$.

We use here that by "Top loci", the center $Z$ is contained in $W_{i}$ locally at points of $W$ for all $i \geq d$, so that transforms of ideals in $W_{i}$ are well defined.

If both $K_{i+1}$ and $K_{i+1}^{\prime}$ are not bold regular or 1, the equality of coefficient ideals implies that $J_{i}^{\prime}=J_{i}^{!}$on $T_{i+1}^{\prime}$. If $K_{i+1}^{\prime}$ is bold regular or 1 , the equality implies the same for $K_{i+1}$, because coefficient ideals in a hypersurface of weak maximal contact are zero if and only if the ideal is bold regular or 1 . If $K_{i+1}=1$ then $K_{i+1}^{\prime}=K_{i+1}^{\curlyvee}=1$ on $T_{i+1}^{\prime}$ and $J_{i}^{\prime}=J_{i}^{!}=1$. If $K_{i+1}$ is bold regular and different 1 , then $o_{i+1}>0$ by definition of $K_{i+1}$. Therefore $m_{i+1}=0$ and $i_{a}\left(\mathcal{M}_{i}\right)=i_{a}(1)=0$ so that $Z$ equals the support of $K_{i+1}$. Here $\mathcal{M}_{i}$ denotes the mobile $\left(J_{i}, c_{i+1}, D_{i}, \ldots, D_{1}, E_{i}, \ldots, E_{1}\right)$ in $W$. Then $K_{i+1}^{\prime}$ equals 1 and $a^{\prime}$ lies outside $T_{i+1}^{\prime}$. This shows that in all cases $J_{i}^{\prime}=J_{i}^{!}$on $T_{i+1}^{\prime}$.

The definition of $M_{i}^{\prime}=I_{W_{i}^{\prime}}\left(D_{i}^{\prime} \cap W_{i}^{\prime}\right)$ with $D_{i}^{\prime}$ as in "Transform of mobile" implies that $J_{i}^{\prime}=M_{i}^{\prime} \cdot I_{i}^{\prime}$ with $I_{i}^{\prime}=I_{i}^{\curlyvee}$ on $T_{i+1}^{\prime}$, and $I_{i}^{\prime}=J_{i}^{\prime}$ outside. Thus property (1) of setups holds for $J_{n}^{\prime}, \ldots, J_{i}^{\prime}$, and (3) and (4) follow from the construction. As for property (2), we may assume by the assertions of "Transversality" applied to $W$ that $W_{i}$ and $Z$ are transversal to $D_{i}$. As $W_{i}^{\prime}=W_{i}^{\curlyvee}$ on $O_{i+1}^{\prime}$ and $D_{i}^{\prime}=\emptyset$ outside $T_{i+1}^{\prime} \subset O_{i+1}^{\prime}$ we conclude that $W_{i}^{\prime}$ is transversal to $D_{i}^{\prime}$. Hence $M_{i}^{\prime}$ is a 
normal crossings divisor in $W_{i}^{\prime}$ at $a^{\prime}$.

The truncated setup $J_{n}^{\prime}, \ldots, J_{i}^{\prime}$ is by construction induced by a locally tuned truncated setup of ${ }^{i} \mathcal{M}^{\prime}$ along top $\left(t_{n}^{\prime}, \ldots, t_{i+1}^{\prime}\right)$. By definition of $P_{i}^{\prime}$ and $E_{i}^{\prime}$ we have $P_{i}^{\prime}=P_{i}^{\curlyvee}$ and $Q_{i}^{\prime}=Q_{i}^{\curlyvee}$ and thus $K_{i}^{\prime}=K_{i}^{\curlyvee}$ on $O_{i}^{\prime}$. This completes the induction step from $i+1$ to $i$.

If $J=M \cdot I$ in $V$ with controlled transform $J^{!}=I\left(Y^{\prime} \cap V^{\prime}\right)^{-c} \cdot J^{*}$ in $V^{\prime}$ and if $M^{\prime}=$ $I\left(Y^{\prime} \cap V^{\prime}\right)^{o-c} \cdot M^{*}$ with $o=\operatorname{ord}_{a} I$ and $I^{\prime}=I^{\curlyvee}=I\left(Y^{\prime} \cap V^{\prime}\right)^{-o} \cdot I^{*}$, we get

$$
\begin{aligned}
J^{\prime} & =I\left(Y^{\prime} \cap V^{\prime}\right)^{-c} \cdot J^{*}= \\
& =I\left(Y^{\prime} \cap V^{\prime}\right)^{-c} \cdot(M \cdot I)^{*}= \\
& =I\left(Y^{\prime} \cap V^{\prime}\right)^{-c} \cdot M^{*} \cdot I^{*}= \\
& =I\left(Y^{\prime} \cap V^{\prime}\right)^{-c} \cdot I\left(Y^{\prime} \cap V^{\prime}\right)^{c-o} \cdot M^{\prime} \cdot I\left(Y^{\prime} \cap V^{\prime}\right)^{o} \cdot I= \\
& =M^{\prime} \cdot I^{\prime} .
\end{aligned}
$$

We have the following formulas on $O_{i}^{\prime}$. As $M_{i}^{\prime}=M_{i}^{*} \cdot I_{W_{i}^{\prime}}\left(Y^{\prime} \cap W_{i}^{\prime}\right)^{o_{i}-c_{i+1}}$ and $c_{i+1}^{\prime}=c_{i+1}$ and $o_{i}^{\prime}=o_{i}$ we get

$$
\begin{aligned}
P_{i}^{\curlyvee} & =\left(I_{i}+M_{i}^{o_{i} / c_{i+1}-o_{i}}\right)^{\curlyvee}= \\
& =I_{i}^{\curlyvee}+\left(M_{i}^{\prime}\right)^{o_{i} / c_{i+1}-o_{i}}= \\
& =I_{i}^{\curlyvee}+\left(M_{i}^{\prime}\right)^{o_{i} / c_{i+1}-o_{i}}= \\
& =I_{i}^{\prime}+\left(M_{i}^{\prime}\right)^{o_{i}^{\prime} / c_{i+1}^{\prime}-o_{i}^{\prime}}= \\
& =P_{i}^{\prime} .
\end{aligned}
$$

On the other hand, $P_{i+1}^{\prime}=P_{i+1}^{\curlyvee}$ on $O_{i+1}^{\prime}$ and thus $W_{i}^{\prime}=W_{i}^{\curlyvee}$. As $E_{i}^{\prime}=E_{i}^{\curlyvee}$ on $O_{i}^{\prime}$ we get, setting $r_{i}=\operatorname{ord}_{a} Q_{i}$,

$$
\begin{aligned}
Q_{i}^{\curlyvee} & =\left(I_{W_{i}}\left(E_{i} \cap W_{i}\right)\right)^{\curlyvee}= \\
& =\left(I_{W_{i}}\left(E_{i} \cap W_{i}\right)\right)^{*} \cdot I_{W_{i}^{\prime}}\left(Y^{\prime} \cap W_{i}^{\prime}\right)^{-r_{i}}= \\
& =\left(I_{W_{i}^{\prime}}\left(E_{i}^{*} \cap W_{i}^{\prime}\right) \cdot I_{W_{i}^{\prime}}\left(Y^{\prime} \cap W_{i}^{\prime}\right)^{-r_{i}}=\right. \\
& =I_{W_{i}^{\prime}}\left(E_{i}^{\prime} \cap W_{i}^{\prime}\right)= \\
& =Q_{i}^{\prime} .
\end{aligned}
$$

The superscript ${ }^{*}$ in the second line above denotes the total transform of $I_{W_{i}}\left(E_{i} \cap W_{i}\right)$ under the blowup $W_{i}^{\prime} \rightarrow W_{i}$, whereas in the third line it denotes the total transform of $E_{i}$ under $W^{\prime} \rightarrow W$

Observe that if $o_{i}^{\prime}=\operatorname{ord}_{a^{\prime}} I_{i}^{\prime}=\operatorname{ord}_{a^{\prime}} I_{i}^{\curlyvee}$ is smaller than $o_{i}$ and if $M_{i}$ appears in $P_{i}$ (i.e., if $\left.0<o_{i}<c_{i+1}\right)$ then $M_{i}^{\prime}=M_{i}^{*} \cdot I_{W_{i}^{\prime}}\left(Y^{\prime} \cap W_{i}^{\prime}\right)^{o_{i}-c_{i+1}} \neq M_{i}^{*} \cdot I_{W_{i}^{\prime}}\left(Y^{\prime} \cap W_{i}^{\prime}\right)^{o_{i}^{\prime}-c_{i+1}^{\prime}}$, though still $M_{i}^{\prime}=I_{W_{i}^{\prime}}\left(D_{i}^{\prime} \cap W_{i}^{\prime}\right)$ and $J_{i}^{\prime}=M_{i}^{\prime} \cdot I_{i}^{\prime}$. Hence $P_{i}^{\prime}$ and $K_{i}^{\prime}$ are in this case not the weak transforms of $P_{i}$ and $K_{i}$. In particular, $W_{i-1}^{\prime}$ will not be the weak transform of $W_{i-1}$. Hence $E_{i}^{\prime}$ must then equal the whole exceptional locus minus $E_{n}^{\prime}+\ldots+E_{i+1}^{\prime}$ to have the center transversal to the exceptional locus.

\section{Decrease of invariant}

Let $\mathcal{M}^{\prime}=\left(\mathcal{J}^{\prime}, c^{\prime}, D^{\prime}, E^{\prime}\right)$ be the transform of $\mathcal{M}=(\mathcal{J}, c, D, E)$ by blowing up $Z=\operatorname{top}(i(\mathcal{M}))$ in $W$. We show that $i_{a^{\prime}}\left(\mathcal{M}^{\prime}\right)<i_{a}(\mathcal{M})$ for $a \in Z, a^{\prime} \in Y^{\prime}$ and $\mathcal{J} \neq 1<11>$. 
By "Top loci", the center $Z$ is contained for all $i$ in $\operatorname{top}\left(I_{i}\right)$ and $\operatorname{top}\left(K_{i}\right)$. This implies for points $a^{\prime}$ in $W_{i}^{\prime}$ above $a$ that $\operatorname{ord}_{a^{\prime}} I_{i}^{\curlyvee} \leq \operatorname{ord}_{a} I_{i}$ and $\operatorname{ord}_{a^{\prime}} K_{i}^{\curlyvee} \leq \operatorname{ord}_{a} K_{i}$. From "Commutativity" follows that $I_{i}^{\prime}=I_{i}^{\curlyvee}$ and hence $o_{i}^{\prime} \leq o_{i}$ on $T_{i+1}^{\prime}$, and $K_{i}^{\prime}=K_{i}^{\curlyvee}$ with $k_{i}^{\prime} \leq k_{i}$ on $O_{i}^{\prime}$. Combining these inequalities gives by descending horizontal induction for all points $a^{\prime}$ of $W^{\prime}$ and for $d$ maximal with $o_{d}=0$ that $\left(t_{n}^{\prime}, \ldots, t_{d+1}^{\prime}\right) \leq\left(t_{n}, \ldots, t_{d+1}\right)$.

If $d=0$ or if $d \geq 1$ and $m_{d}=(0,0)$ the center equals locally the support of $K_{d+1}$. Hence $K_{d+1}^{\prime}=K_{d+1}^{r}=1$ and $k_{d+1}^{\prime}=0<k_{d+1}$ on $O_{d+1}^{\prime}$. If $d \geq 1$ and $m_{d} \neq(0,0)$, a computation in local coordinates using the definition of maximal tight shortcuts shows that $m_{d}^{\prime}<m_{d}$ on $T_{d+1}^{\prime}<11>$. In both cases we get $i_{a^{\prime}}\left(\mathcal{M}^{\prime}\right)<$ $i_{a}(\mathcal{M})$.

\section{Transversality}

Let $\mathcal{M}=(\mathcal{J}, c, D, E)$ be the mobile obtained at a certain stage of the resolution process. We show that $D_{i}, E_{i}$ and $|E|$ are normal crossings divisors in $W$ transversal to $Z$, and that $|E|$ coincides with the exceptional locus accumulated so far. And, if $W_{n}, \ldots, W_{1}$ is the local flag of a punctual setup $J_{n}, \ldots, J_{1}$ of $\mathcal{M}$ at $a$ as constructed in "Commutativity", $W_{i}$ is transversal to $D_{i}$ and $E_{i}, \ldots, E_{1}$, and not contained in $E_{i}+\ldots+E_{1}$ for all $i<12>$.

The divisors $E_{d}, \ldots, E_{1}$ are irrelevant for the definition of $K_{d}, \ldots, K_{1}$. But it is necessary to know that $W_{d}$ is transversal to $E_{d}, \ldots, E_{1}$ in order to have $Z=\operatorname{top}\left(N_{d}\right) \subset W_{d}$ transversal to $|E|$. We have $T_{i}=\emptyset$ locally at $a$ for some $i \geq d$ by "Decrease of invariant", hence $O_{d-1}=\emptyset$ if $d \geq 2$ and then $|E|=E_{n}+\ldots+E_{d-1}$. Note that $E_{d-1}$ may be non empty because $O_{d}$ need not be empty when $T_{d}=\emptyset$. If $d=1$, then $|E|=E_{n}+\ldots+E_{1}$ by vertical induction and definition of $E_{1}$.

Assume by vertical induction that these properties hold at the prior stages of the process. The definition of $D_{i}$ and $E_{i}$ in "Transform of mobile" implies, by the persistence of normal crossings under blowup in transversal centers, that $D_{i}, E_{i}$ and $|E|$ are normal crossings divisors. It has been proven in "Decrease of invariant" that some $T_{j}$ is always empty. If $j \geq 2$ then $O_{j-1}=\emptyset$. The definition of $E_{j-1}$ if $j \geq 2$ and of $E_{1}$ if $j=1$ implies by vertical induction that $|E|$ coincides with the exceptional locus. We have $|E|=E_{n}+\ldots+E_{j}$ and $E_{j-1}=\ldots=E_{1}=\emptyset$ outside $O_{j}$.

We show that $W_{i}$ is transversal to $D_{i}$ and $E_{i}, \ldots, E_{1}$ for all $i$. Let $W \rightarrow W^{\circ}$ denote the last blowup. By the induction hypothesis, $W_{i}^{\circ}$ is transversal to $D_{i}^{\circ}$ and $E_{i}^{\circ}, \ldots, E_{1}^{\circ}$, and $Z^{\circ}$ is transversal to $D_{i}^{\circ}$ and $\left|E^{\circ}\right|$. Recall that $W_{i}=\left(W_{i}^{\circ}\right)^{\curlyvee}$ on $O_{i+1}$, that $D_{i}=\emptyset$ outside $T_{i+1}$ and $E_{i}=\ldots=E_{1}=\emptyset$ outside $O_{i+1}$. This implies that $W_{i}$ is transversal to $D_{i}$ and $E_{i}, \ldots, E_{1}$.

The transversality of $W_{i}$ with $D_{i}$ need not follow from the transversality of $W_{i}$ with $E_{i}$ since $D_{i}$ could contain other exceptional components than $E_{i}$, and because $W_{i}$ will in general not be transversal to $|E|$. The transversality of $W_{i}$ with $D_{i}$ is needed in order to know that the $M_{i}$ are normal crossings divisors in $W_{i}$. 
The fact that $W_{i}$ is transversal to $E_{i}$ is needed to know that $\operatorname{ord}_{a} Q_{i}$ equals $\operatorname{ord}_{a} E_{i}$ and is thus independent of $W_{i}$. The transversality of $W_{d}$ with $E_{d}$ is needed to know that $Z$ is transversal with $|E|$, which in turn implies that $\left|E^{\prime}\right|$ and all $D_{i}^{\prime}$ are normal crossings divisors. Observe that $W_{i}$ will in general not be transversal to $E_{i+1}$.

We show that $W_{i}$ is not contained in $E_{i}+\ldots+E_{1}$. Assume that this holds in $W^{\circ}$. Inside $O_{i+1}$ we have $W_{i}=\left(W_{i}^{\circ}\right)^{\curlyvee}$, and $E_{i}+\ldots+E_{1}$ is either the weak transform of $E_{i}^{\circ}+\ldots+E_{1}^{\circ}$ or its union with $Y$. Hence, if $Z^{\circ}$ is strictly contained in $W_{i}^{\circ}, W_{i}$ is not contained in $E_{i}+\ldots+E_{1}$ inside $O_{i+1}$. If $Z^{\circ}=W_{i}^{\circ}$ we have $J_{i}^{\circ}=1$ because $Z^{\circ} \subset \operatorname{top}\left(J_{i}^{\circ}\right)$ by "Top loci". Therefore $K_{i+1}^{\circ}$ and hence $P_{i+1}^{\circ}$ are bold regular or 1 , with $Z^{\circ}=W_{i}^{\circ}$ the support of $P_{i+1}^{\circ}$. Then $o_{i+1}=0<o_{i+1}^{\circ}$, so we are outside $O_{i+1}$. As $E_{i}, \ldots, E_{1}$ are empty there, $W_{i}$ is not contained in $E_{i}+\ldots+E_{1}$ also in this case.

The fact that $W_{i}$ is not contained in $E_{i}$ for $i>d$ is needed to have $Q_{i} \neq 0$ and hence $\operatorname{top}\left(K_{i}\right)=\operatorname{top}\left(P_{i}\right) \cap \operatorname{top}\left(Q_{i}\right)$. For $i=d$ it is not needed since $K_{d}=1$.

We show that $Z$ is transversal to $D_{i}, E_{i}$ and $|E|$. As $|E|$ equals the exceptional locus the $D_{i}$ are supported by certain components of $|E|$. Let $d$ be the largest index with $o_{d}=0$ at $a$, setting $d=0$ if all $o_{i}>0$. By "Top loci", $Z$ equals locally top $\left(o_{d}, k_{d}, m_{d}\right)=\operatorname{top}\left(N_{d}\right)$ if $d \geq 1$, and $\{a\}$ otherwise. As $N_{d}$ is a shortcut of $M_{d}=I_{W_{d}}\left(D_{d} \cap W_{d}\right)$ and $W_{d}$ is transversal to $E_{d}, \ldots, E_{1}$ we see that $Z$ is transversal to $E_{d}, \ldots, E_{1}$. For $i>d$ we have $I_{i} \neq 1$ and $Q_{i} \neq 0$ so that $\operatorname{top}\left(K_{i}\right)=$ $\operatorname{top}\left(P_{i}\right) \cap \operatorname{top}\left(Q_{i}\right)$. As $Z \subset \operatorname{top}\left(K_{i}\right)$ by "Top loci" we get $Z \subset \operatorname{top}\left(P_{i}\right) \cap \operatorname{top}\left(E_{i}\right)$. Therefore $Z$ is contained for $i>d$ in all components of $E_{i}$ it meets. This proves that $Z$ is transversal to $|E|$, hence also to all $D_{i}$ and $E_{i}$.

\section{Independence and semicontinuity}

Suppose that we are given, locally on $W$, a truncated tuned setup $\mathcal{J}_{n}, \ldots, \mathcal{J}_{i}$ of a mobile $(\mathcal{J}, c, D, E)$ with truncated invariant $\left(t_{n}, \ldots, t_{i}\right)$. We prove by descending horizontal induction that $\left(t_{n}, \ldots, t_{i}\right)$ is upper semicontinuous on $W$ and independent of the choice of the setup.

In case $i=n$, the tag $t_{n}=\left(o_{n}, k_{n}, m_{n}\right)$ is constructed without choices in terms of the
stalk $J_{n}$ of $\mathcal{J}$, the control $c_{n+1}$ and the divisors $D_{n}$ and $E_{n}$. Its first component $o_{n}$ is
upper semicontinuous since it is the order of a coherent ideal. The definition of $P_{n}$ and the
coherence of $D_{n}$ imply that the order of $P_{n}$ is upper semicontinuous. As also $E_{n}$ is coherent,
the order $k_{n}$ of $K_{n}=P_{n} \cdot Q_{n}$ is upper semicontinuous. The maximal tight shortcut $N_{n}$ of
$M_{n}$ has by construction a tag $m_{n}$ which is upper semicontinuous. Hence $t_{n}=\left(o_{n}, k_{n}, m_{n}\right)$
is upper semicontinuous.

Assume that the assertions have been proven for $n, \ldots, i+1$. Fix a point $b \in$ $W$. Locally, $\left(t_{n}, \ldots, t_{i+1}\right)$ attains by semicontinuity at $b$ its maximum. Choose closed subschemes $W_{n}, \ldots, W_{i+1}$ of a neighborhood $U$ of $b$ where $\mathcal{J}_{n}, \ldots, \mathcal{J}_{i+1}$ are defined and induce at all points of $U \cap \operatorname{top}\left(t_{n}, \ldots, t_{i+1}\right)$ a truncated punctual setup of $\mathcal{J}$.

After shrinking $U$ there exists a closed hypersurface $W_{i}$ in $W_{i+1}$ which has weak 
maximal contact with $K_{i+1}$ at all points $a$ of top $\left(K_{i+1}\right)$. By "Top loci" we know that if $o_{i+1}>0$ then $\operatorname{top}\left(t_{n}, \ldots, t_{i+1}\right)=\operatorname{top}\left(K_{i+1}\right)$. By "Order of coefficient ideals", $\operatorname{ord}_{a} J_{i}$ depends only on the locus top $\left(K_{i+1}\right)$ and its transforms under the blowups constructed there. It is hence independent of the choice of the local flag. The same then holds for $o_{i}=\operatorname{ord}_{a} J_{i}-\operatorname{ord}_{a} M_{i}$ because $\operatorname{ord}_{a} M_{i}=\operatorname{ord}_{a} D_{i}$ by "Transversality".

If $o_{i}>0$, then $K_{i}=P_{i} \cdot Q_{i}$ and $k_{i}=o_{i}+\operatorname{ord}_{a} Q_{i}=o_{i}+\operatorname{ord}_{a} E_{i}$ because $W_{i}$ is transversal to $E_{i}$. If $o_{i}=0$, then $k_{i}=0$ and $m_{i}$ only depends on $D_{i}$ and $c_{i+1}$. The independence and the upper semicontinuity of $t_{i}=\left(o_{i}, k_{i}, m_{i}\right)$ follow in both cases.

\section{Order of coefficient ideals}

Let $V^{\circ}$ be a closed regular subscheme of a regular scheme $W^{\circ}$ and let $K^{\circ}$ be a coherent ideal in $W^{\circ}$ with coefficient ideal $J^{\circ}$ in $V^{\circ}$. We prove that the order of $J^{\circ}$ in $V^{\circ}$ is determined by the locus top $\left(K^{\circ}\right) \cap V^{\circ}$ and its transforms under certain blowups $<13>$.

Fix a point $a^{\circ}$ in $\operatorname{top}\left(K^{\circ}\right) \cap V^{\circ}$ and set $c=\operatorname{ord}_{a^{\circ}} K^{\circ}, e=\operatorname{ord}_{a^{\circ}} J^{\circ}$. Let $K$ and $J$ denote the ideals generated by $K^{\circ}$ and $J^{\circ}$ in $W=W^{\circ} \times \mathbb{A}^{1}$ and $V=V^{\circ} \times \mathbb{A}^{1}$. Then $J$ is the coefficient ideal of $K$ in $V$. We have $\operatorname{top}(J, c) \subset \operatorname{top}(K) \cap V$. Set $L=\left\{a^{\circ}\right\} \times \mathbb{A}^{1} \subset V$ and $a=\left(a^{\circ}, 0\right)$. Consider the blowup $W^{\prime} \rightarrow W$ of $W$ with center $a$ and exceptional locus $Y^{\prime}$. Let $K^{\prime}$ and $J^{\prime}$ be the weak and controlled transform of $K$ and $J$ with respect to $c$. From $c^{\prime}=c$ at all points of $Y^{\prime}$ follows that $J^{\prime}$ is the coefficient ideal of $K^{\prime}$ in $V^{\prime}=V^{\curlyvee}$, so that $\operatorname{top}\left(J^{\prime}, c\right) \subset \operatorname{top}\left(K^{\prime}, c\right) \cap V^{\prime}$.

We have $J^{\prime}=I^{\prime} \cdot I_{V^{\prime}}\left(Y^{\prime} \cap V^{\prime}\right)^{e-c}$ for some ideal $I^{\prime}$ in $V^{\prime}$. Let $L^{\prime}$ be the weak transform of $L$. Since the order of $K$ along $L$ is $c$, the order of $K^{\prime}$ along $L^{\prime}$ is also $c$. Set $a^{\prime}=L^{\prime} \cap Y^{\prime}$. We blow up $W^{\prime}$ with center $a^{\prime}$. Continuing this procedure we get, after $k$ steps, a morphism $W^{k} \rightarrow W^{k-1}$ with exceptional locus $Y^{k}$. The ideals $K^{k}$ and $J^{k}$ are the weak and controlled transforms of $K^{k-1}$ and $J^{k-1}$ and $J^{k}$ is the coefficient ideal of $K^{k}$. Thus top $\left(J^{k}, c\right) \subset \operatorname{top}\left(K^{k}\right) \cap V^{k}$. Then the weak transform $I^{k}$ of $J^{k-1}$ in $V^{k}$ satisfies $J^{k}=I^{k} \cdot I_{V^{k}}\left(Y^{k} \cap V^{k}\right)^{k(e-c)}$.

Let $L^{k}$ be the weak transform of $L^{k-1}$. The order of $K^{k}$ along $L^{k}$ is $c$. We set $a^{k}=L^{k} \cap Y^{k}$. Note that $Y^{k} \cap V^{k} \subset \operatorname{top}\left(J^{k}, c\right)$ if and only if $k(e-c) \geq c$. For each $k$, let now $Y^{k} \cap V^{k}$ be the center of the next blowup. The associated morphism $W^{k+1} \rightarrow W^{k}$ induces an isomorphism $V^{k+1} \rightarrow V^{k}$ and $J^{k+1}=J^{k} \cdot I\left(Y^{k} \cap V^{k}\right)^{-c}$. These blowups with center $Y^{k} \cap V^{k}$ can be repeated $p_{k}$ times where $p_{k}$ is the integral part of $\frac{k(e-c)}{c}$. Note that $p_{k}$ depends only on the locus $\operatorname{top}\left(K^{\circ}\right) \cap V^{\circ}$ together with its transforms under the previous blowups and on the control $c$. The assertion then follows from $\operatorname{ord}_{a}\left(J^{\circ}\right)=\left(1+\lim _{k \mapsto \infty} \frac{p_{k}}{k}\right) \cdot c$.

\section{Resolution of mobiles}

Let $\mathcal{M}=(\mathcal{J}, c, D, E)$ be any mobile in $W$ with ideal $\mathcal{J}$ and control $c$ in $V$ which admits locally on $W$ tuned setups with local flags given by hypersurfaces $W_{i-1}$ 
in $W_{i}$ which are osculating for $P_{i}$. Such setups exist e.g. when the combinatorial handicap $D$ is empty, independently of the transversal handicap. We show that $\mathcal{M}$ has a strong resolution. For this we may assume that $c \leq$ the supremum of the order of $\mathcal{J}$ in $V$. Blow up $W$ in $Z=\operatorname{top}(i(\mathcal{M}))$ with transformed mobile $\mathcal{M}^{\prime}=\left(\mathcal{J}^{\prime}, c^{\prime}, D^{\prime}, E^{\prime}\right)$ in $W^{\prime}$. Then $\mathcal{M}^{\prime}$ admits by "Commutativity" locally tuned setups as before. We have seen in "Decrease of invariant" that $i_{a^{\prime}}\left(\mathcal{M}^{\prime}\right)<i_{a}(\mathcal{M})$ for $\mathcal{J} \neq 1$ and for all points $a \in Z$ and $a^{\prime} \in Y^{\prime}$. As the invariant takes values in a well ordered set vertical induction applies. Hence finitely many blowups make the order of the transform of $\mathcal{J}$ drop below $c$. By transversality, all centers are transversal to the exceptional loci. Equivariance follows from property (a) of the invariant. This establishes the strong resolution of mobiles.

\section{Resolution of schemes}

The resolution of mobiles is used to construct a strong resolution of reduced singular subschemes $X$ of $W^{<14>}$. We may assume that $X$ is different from $W$, and that $W$ is equidimensional. Let $\mathcal{J}$ be the ideal of $X$ in $W$. Associate to it the mobile $\mathcal{M}=(\mathcal{J}, c, D, E)$ with control $c=1$ and empty handicaps. At any stage $W^{\prime}$ of the resolution of $\mathcal{M}$ the controlled transform $\mathcal{J}^{\prime}$ of $\mathcal{J}$ defines a subscheme of $W^{\prime}$ formed by the strict transform $X^{\prime}$ of $X$ and some components inside the exceptional locus.

As the final controlled transform of $\mathcal{J}$ equals 1 , there corresponds to each component of $X$ a stage where the strict transform of the component has become regular and has been taken locally as the center of the next blowup. Let $X_{1}$ be those components of $X$ which reach this stage first. The corresponding strict transform $X_{1}^{\prime}$ of $X_{1}$ is regular and, by "Transversality" and property (d) of the invariant, transversal to the exceptional locus.

Write $X^{\prime}=X_{1}^{\prime} \cup X_{2}^{\prime}$ with $X_{2}^{\prime}$ the strict transform of the remaining components of $X$. Stop here the resolution process of the mobile $(\mathcal{J}, c, D, E)$ and define a new mobile whose resolution implies the separation of $X_{2}^{\prime}$ from $X_{1}^{\prime}$. Omitting primes, let $\mathcal{K}$ be the ideal of $X_{2}$ in $W$. Let $\mathcal{J}$ be the coefficient ideal of $\mathcal{K}$ in $X_{1}$ with control $c$ the maximum on $X_{1}$ of the order of $\mathcal{K}$ in $W$. Set all $D_{i}$ and $E_{i}$ empty with the exception of $E_{n}$, where $n$ is the dimension of $X_{1}$, for which we take the exceptional locus produced so far.

Resolve the mobile $(\mathcal{J}, c, D, E)$. The controlled transforms of $\mathcal{J}$ are the coefficient ideals of the weak transforms of $\mathcal{K}$ as long as the maximum of the order of $\mathcal{K}$ in $W$ along $X_{1}$ remains constant. Therefore the resolution of $(\mathcal{J}, c, D, E)$ will make this maximum drop. Hence also the maximum of the order of the strict transform of $\mathcal{K}$ in $W$ along $X_{1}$ drops. Iterating this process the final strict transform $X_{2}^{\prime}$ of $X_{2}$ will be separated from the weak transform $X_{1}^{\prime}$ of $X_{1}$. Now induction on the number of components applies to construct a sequence of blowups which makes $X_{2}^{\prime}$ regular and transversal to the transversality locus. Thus $X$ has become regular.

Embeddedness and equivariance follow from the resolution of mobiles. The resolution of $X$ does not depend on the embedding of $X$ since, under embeddings 
of $W$ into some $W^{+}$, the restriction of $i\left(\mathcal{M}^{+}\right)$to $W$ equals $i(\mathcal{M})$. This proves excision. The construction of a strong resolution of $X$ is completed.

\section{Appendix}

We indicate some references where concepts and arguments of this paper have their origins or analogues.

(1) Maximal tight shortcut: Standard construction to prove combinatorial resolution, cf. [EV1] 2, p. 114, [BM3] 6.16(b), p. 260.

(2) Mobile: Including handicaps extends and refines [H2] 1.1, p. 54, [A1] 3, p. 90, 13, p. 208, [EV1] 1.2, p. 112, [BM3] 4.1, p. 241, [BS], p. 407. For the combinatorial handicap cf. [EV1] 3.1, p. 116, and 4.20, p. 127, [BM3] 4.23, p. 247, for the transversal handicap cf. [A1] 2, p. 89, 5, p. 98, [EV1] 6.17, p. 149, [BM3] 6.8, p. 256.

(3) Controlled transform: Suitable transform to have coefficient ideals commute with blowup, cf. [H2] 1.10, p. 57, [EV1] 1.4, p. 113, [BM3] 4.4, p. 242.

(4) Composition ideal: Cf. [EV1] 6.20.1, p. 153, 6.21.1, p. 154, 6.21.2, p. 155, [BM3] 4.23, p. 247.

(5) Coefficient ideal: Transfers the resolution problem to smaller dimension, $\mathrm{cf}$. [H2] 8.5.4, p. 112, [A2] 7.1, p. 19, [EV1] 4.14, p. 122, [BM3] 4.18, p. 246.

(6) Weak maximal contact: Characteristic free notion to make the order of the coefficient ideal coordinate independent, cf. [A2] 1.6, p. 6, [H2], 2.4, p. 63, $[\mathrm{AHV}]$ p. 6. Osculating hypersurfaces are a characteristic zero construction to guarantee weak maximal contact persistent under blowup, cf. [A1] 13.5, p. 211, [H2] 8.2, p. 106, 8.4, p. 108, [AHV] 1.2.5.7, p. 34, [EV1] 4.4, p. 118, 4.11, p. 121, [BM3] 4.12, p. 244.

(7) Setup: References as for composition and coefficient ideal. In contrast to [H2], 1.1, p. 54, 2.3, p. 62, 2.6. and 2.7, p. 67, [EV1] 5.1, p. 129, 5.11, p. 131, [BM3] 4.19, p. 246, patchings and equivalence relations are not needed.

(8) Invariant: Cf. [EV1] 4.16, p. 124, 4.20, p. 127, 6.11, p. 140, 6.13, p. 143 [BM3] 4.20, p. 246, 6.15, p. 259.

(9) Transform of mobile: Distinguishes old and new exceptional components, cf. [A1] 2, p. 89, 5, p. 98 , [EV1] 1.4, p. 113, 6.17, p. 149, [BM3] 4.4, p. 242, 6.8 , p. 256.

(10) Commutativity: Needed to construct setups of the transformed mobile, cf. [G] 3.11, p. 309, [H2] 8.5.6, p. 113, [EV1] 4.6, p. 119, 4.15, p. 123, [BM3] 4.19 , p. 246, 4.24, p. 248.

(11) Decrease of invariant: Cf. [A1], 16.5, p. 225, 17.4, p. 233, [H1], p. 312, [EV1] 6.13 , p. 143, [BM3], p. 260.

(12) Transversality: Cf. [EV1] step 6.2, p. 154, [BM3] 4.12, p. 244.

(13) Order of coefficient ideals: The argument is from [H2] 2.8, p. 68, cf. also [A1] 7.4, p. 141. 
(14) Resolution of schemes: Cf. [EV2] 1.5.

\section{References}

[A1] S. Abhyankar, Good points of a hypersurface, Adv. Math. 68 (1988), 87-256.

[A2] S. Abhyankar, Desingularization of plane curves, in: Algebraic Geometry, Arcata 1981, Proc. Symp. Pure Appl. Math. 40, Amer. Math. Soc.

[AHV] J.-M. Aroca, H. Hironaka and J.-L. Vicente, The theory of the maximal contact, Mem. Mat. Inst. Jorge Juan Madrid 29 (1975).

[AJ] D. Abramovich and J. de Jong, Smoothness, semi-stability and toroidal geometry, J. Alg. Geometry 6 (1997), 789-801.

[AW] D. Abramovich and J. Wang, Equivariant resolution of singularities in characteristic 0, Math. Res. Letters 4 (1997), 427-433.

[BM1] E. Bierstone and P. Milman, Uniformization of analytic spaces, J. Amer. Math. Soc. 2 (1989), 801-836.

[BM2] E. Bierstone and P. Milman, Semianalytic and subanalytic sets, Publ. Math. IHES 67 (1988), 5-42.

[BM3] E. Bierstone and P. Milman, Canonical desingularization in characteristic zero by blowing up the maximum strata of a local invariant, Invent. math. 128 (1997), 207-302.

[BP] F. Bogomolov and T. Pantev, Weak Hironaka Theorem, Math. Res. Letters 3 (1996), 299-307.

[BS] G. Bodnár and J. Schicho, Automated Resolution of Singularities for Hypersurfaces, J. Symb. Comp. 30 (2000), 401-428.

[BV] A. Bravo and O. Villamayor, A strengthening of resolution of singularities in characteristic zero, to appear in Proc. London Math. Soc.

[EV1] S. Encinas and O. Villamayor, Good points and constructive resolution of singularities, Acta Math. 181 (1998), 109-158.

[EV2] S. Encinas and O. Villamayor, A proof of desingularization over fields of characteristic zero, http://arxiv.org/abs/math.AG/0101208

[G] J. Giraud, Sur la théorie du contact maximal, Math. Z. 137 (1974), 285-310.

[Ha1] H. Hauser, Resolution of singularities 1860-1999, in: Hauser et al. (ed.), Resolution of Singularities Progress in Math. 181, Birkhäuser, 2000.

[Ha2] H. Hauser, The Hironaka Theorem on Resolution of Singularities (or: A proof we always wanted to understand), to appear in Bull Amer. Math. Soc.

[H1] H. Hironaka, Resolution of singularities of an algebraic variety over a field of characteristic zero, Ann. Math. 79 (1964), 109-326.

[H2] H. Hironaka, Idealistic exponents of singularity, in: Algebraic Geometry, 52-125, The Johns Hopkins Centennial Lectures, 1977.

[V1] O. Villamayor, Constructiveness of Hironaka's resolution, Ann. Sci. Éc. Norm. Sup. Paris 22 (1989), 1-32.

[V2] O. Villamayor, Patching local uniformizations, Ann. Scient. Éc. Norm. Sup. Paris 25 (1992), 629-677.

Santiago Encinas

Universidad de Valladolid

E-47014 Spain

e-mail: sencinas@maf.uva.es
Herwig Hauser

Universität Innsbruck

A-6020 Austria

e-mail: herwig.hauser@uibk.ac.at

(Received: April 30, 2002) 